\title{
Search for microscopic black hole signatures at the Large Hadron Collider is
}

\author{
CMS Collaboration *
}

CERN, Switzerland

\section{A R T I C L E I N F O}

\section{Article history:}

Received 15 December 2010

Received in revised form 5 February 2011

Accepted 13 February 2011

Available online 15 February 2011

Editor: M. Doser

\section{Keywords:}

CMS

Physics

Black holes

Extra dimensions

\begin{abstract}
A B S T R A C T
A search for microscopic black hole production and decay in pp collisions at a center-of-mass energy of $7 \mathrm{TeV}$ has been conducted by the CMS Collaboration at the LHC, using a data sample corresponding to an integrated luminosity of $35 \mathrm{pb}^{-1}$. Events with large total transverse energy are analyzed for the presence of multiple high-energy jets, leptons, and photons, typical of a signal expected from a microscopic black hole. Good agreement with the standard model backgrounds, dominated by QCD multijet production, is observed for various final-state multiplicities and model-independent limits on new physics in these final states are set. Using simple semi-classical approximation, limits on the minimum black hole mass are derived as well, in the range $3.5-4.5 \mathrm{TeV}$. These are the first direct limits on black hole production at a particle accelerator.
\end{abstract}

(C) 2011 CERN. Published by Elsevier B.V. Open access under CC BY-NC-ND license.
One of the exciting predictions of theoretical models with extra spatial dimensions and low-scale quantum gravity is the possibility of copious production of microscopic black holes in particle collisions at the CERN Large Hadron Collider (LHC) [1,2]. Models with low-scale gravity are aimed at solving the hierarchy problem, the puzzlingly large difference between the electroweak and Planck scales.

In this Letter we focus on microscopic black hole production in a model with large, flat, extra spatial dimensions, proposed by Arkani-Hamed, Dimopoulos, and Dvali, and referred to as the ADD model [3,4]. This model alleviates the hierarchy problem by introducing $n$ extra dimensions in space, compactified on an $n$ dimensional torus or sphere with radius $r$. The multidimensional space-time is only open to the gravitational interaction, while the gauge interactions are localized on the $3+1$ space-time membrane. As a result, the effective gravitational coupling is enhanced at distances smaller than $r$, and Newton's law of gravitation is modified at short distances. The "true" Planck scale in $4+n$ dimensions $\left(M_{D}\right)$ is consequently lowered to the electroweak scale, much smaller than the apparent Planck scale of $M_{\mathrm{Pl}} \sim 10^{16} \mathrm{TeV}$ seen by a $3+1$ space-time observer. The relationship between $M_{D}$ and $M_{\mathrm{Pl}}$ follows from Gauss's law and is given as $M_{\mathrm{Pl}}^{2}=8 \pi M_{D}^{n+2} r^{n}$, using the Particle Data Group (PDG) definition [5].

Since in the ADD model gravity is enhanced by many orders of magnitude at distances much smaller than $r$, black hole formation in particle collisions could happen at energies greater than $M_{D}$,

\footnotetext{
(c) CERN, for the benefit of the CMS Collaboration.

* E-mail address: cms-publication-committee-chair@cern.ch.
}

rather than $M_{\mathrm{Pl}}$, which is the case for a $3+1$ space-time. Colliding particles would collapse in a black hole if their impact parameter were smaller than approximately the Schwarzschild radius of a black hole with the mass $M_{\mathrm{BH}}$ equal to the total energy accessible in the collision. The Schwarzschild radius of a black hole with mass $M_{\mathrm{BH}}$ embedded in $4+n$ space-time can be found by solving Einstein's general relativity equations and is given by [6-8]:

$r_{S}=\frac{1}{\sqrt{\pi} M_{D}}\left[\frac{M_{\mathrm{BH}}}{M_{D}} \frac{8 \Gamma\left(\frac{n+3}{2}\right)}{n+2}\right]^{\frac{1}{n+1}}$.

The parton-level cross section of black hole production is derived from geometrical considerations and is given by $\sigma \sim \pi r_{S}^{2}$ $[1,2]$. At LHC energies, this cross section can reach $100 \mathrm{pb}$ for $M_{D}$ of $1 \mathrm{TeV}$. The exact cross section cannot be calculated without knowledge of the underlying theory of quantum gravity and is subject to significant uncertainty. It is commonly accepted [1,2] that the minimum black hole mass $M_{\mathrm{BH}}^{\min }$ cannot be smaller than $M_{D}$; although the formation threshold can be significantly larger than this. When a black hole is formed, some fraction of the colliding parton energy may not be trapped within the event horizon and will be emitted in the form of gravitational shock waves, which results in energy, momentum, and angular momentum loss [9-11]. This effect is particularly model-dependent for black hole masses close to $M_{D}$. In general, black holes in particle collisions are produced with non-zero angular momentum, which also affects their properties and production cross section.

Once produced, the microscopic black holes would decay thermally via Hawking radiation [12], approximately democratically (with equal probabilities) to all standard model (SM) degrees of 
freedom. Quarks and gluons are the dominant particles produced in the black hole evaporation $(\sim 75 \%)$ because they have a large number of color degrees of freedom. The remaining fraction is accounted for by leptons, $W$ and $Z$ bosons, photons, and possibly Higgs bosons. Emission of gravitons by a black hole in the bulk space is generally expected to be suppressed [13], although in some models it can be enhanced for rotating black holes for the case of large $n[10,11,14]$. In some models the evaporation is terminated earlier, when the black hole mass reaches $M_{D}$, with the formation of a stable non-interacting and non-accreting remnant [15]. The Hawking temperature for a black hole in $4+n$ space-time is given by $[1,2,7,8]: T_{H}=\frac{n+1}{4 \pi r_{S}}$ (in Planck units $\hbar=$ $c=k_{B}=1$, where $k_{B}$ is the Boltzmann constant) and is typically in the range of a few hundred GeV. The lifetime for such a microscopic black hole is $\sim 10^{-27} \mathrm{~s}[1,2,8]$.

Here we consider semi-classical black holes, whose properties are similar to those for classical black holes described by general relativity and whose mass is close enough to $M_{D}$ so that quantum effects can not be ignored completely. There are also models [1618] of quantum black holes that decay before they thermalize, mainly into two-jet final states. We do not consider this signature here, leaving it for dedicated searches in the dijet channel $[19,20]$.

In what follows, we further assume that the semi-classical approximation, which is strictly valid only for $M_{\mathrm{BH}} \gg M_{D}$, still holds even for the $\mathrm{BH}$ masses as low as $M_{D}$. While we expect that unknown quantum corrections to the black hole production and decay may become very important, if not dominant, for $M_{\mathrm{BH}} \approx M_{D}$, we still use semi-classical approximation as a benchmark due to the lack of a better, quantum model of black hole production and decay.

The microscopic black holes produced at the LHC would be distinguished by high multiplicity, democratic, and highly isotropic decays with the final-state particles carrying hundreds of $\mathrm{GeV}$ of energy. Most of these particles would be reconstructed as jets of hadrons. Observation of such spectacular signatures would provide direct information on the nature of black holes as well as the structure and dimensionality of space-time [1]. Microscopic black hole properties are reviewed in more detail in $[10,11]$.

The search for black holes is based on $\sqrt{s}=7 \mathrm{TeV}$ pp collision data recorded by the Compact Muon Solenoid (CMS) detector at the LHC between March and October 2010, which correspond to an integrated luminosity of $34.7 \pm 3.8 \mathrm{pb}^{-1}$. A detailed description of the CMS experiment can be found elsewhere [21]. The central feature of the CMS detector is the 3.8 T superconducting solenoid enclosing the silicon pixel and strip tracker, the electromagnetic calorimeter (ECAL), and the brass-scintillator hadronic calorimeter (HCAL). For triggering purposes and to facilitate jet reconstruction, the calorimeter cells are grouped in projective towers, of granularity $\Delta \eta \times \Delta \phi=0.087 \times 0.087$ at central rapidities and $0.175 \times 0.175$ in the forward region. Here, the pseudorapidity $\eta$ is defined as $-\ln \left(\tan \frac{\theta}{2}\right)$, where $\theta$ is the polar angle with respect to the direction of the counterclockwise beam, and $\phi$ is the azimuthal angle. Muons are measured in the pseudorapidity window $|\eta|<2.4$ in gaseous detectors embedded in the steel return yoke.

The CMS trigger system consists of two levels. The first level (L1), composed of custom hardware, uses information from the calorimeters and muon detectors to select the most interesting events for more refined selection and analysis at a rate of up to $80 \mathrm{kHz}$. The software-based High Level Trigger (HLT) further decreases the rate to a maximum of $\sim 300 \mathrm{~Hz}$ for data storage. The instantaneous luminosity is measured using information from forward hadronic calorimeters [22].

We use data collected with a dedicated trigger on the total jet activity, $H_{T}$, where $H_{T}$ is defined as the scalar sum of the transverse energies $E_{T}$ of the jets above a preprogrammed threshold.
At L1 this jet $E_{T}$ threshold was $10 \mathrm{GeV}$, and the $H_{T}$ threshold was $50 \mathrm{GeV}$. At HLT, the jet $E_{T}$ threshold varied between 20 and $30 \mathrm{GeV}$, and the $H_{T}$ threshold between 100 and $200 \mathrm{GeV}$. The trigger is fully efficient for the offline analysis selections described below. Energetic electrons and photons are also reconstructed as jets at the trigger level and are thus included in the $H_{T}$ sum.

Jets are reconstructed using energy deposits in the HCAL and ECAL, clustered using a collinear and infrared safe anti- $k_{T}$ algorithm with a distance parameter of 0.5 [23]. The jet energy resolution is $\Delta E / E \approx 100 \% / \sqrt{E[\mathrm{GeV}]} \oplus 5 \%$. Jets are required to pass quality requirements to remove those consistent with calorimeter noise. Jet energies are corrected for the non-uniformity and non-linearity of the calorimeter response, as derived using Monte Carlo (MC) samples and collision data [24]. Jets are required to have $E_{T}>20 \mathrm{GeV}$ before the jet-energy-scale corrections and to have $|\eta|<2.6$. Missing transverse energy $\mathbb{E}_{\mathrm{T}}$ is reconstructed as the negative of the vector sum of transverse energies in the individual calorimeter towers. This quantity is further corrected to account for muons in the event, which deposit little energy in the calorimeters, and for the jet energy scale [25].

Electrons and photons are identified as isolated energy deposits in the ECAL, with a shape consistent with that expected for electromagnetic showers. Photons are required to have no matching hits in the inner pixel detector layers, while electrons are required to have a matching track. Electrons and photons are required to have $E_{T}>20 \mathrm{GeV}$ and to be reconstructed in the fiducial volume of the barrel $(|\eta|<1.44)$ or the endcap $(1.56<|\eta|<2.4)$. The ECAL has an ultimate energy resolution better than $0.5 \%$ for unconverted photons or electrons with transverse energies above $100 \mathrm{GeV}$ [26]. In 2010 collision data, for $E_{T}>20 \mathrm{GeV}$, this resolution is better than $1 \%$ in the barrel.

Muons are required to have matched tracks in the central tracker and the muon spectrometer, to be within $|\eta|<2.1$, be consistent with the interaction vertex to suppress backgrounds from cosmic ray muons, be isolated from other tracks, and have transverse momentum $p_{T}$ above $20 \mathrm{GeV}$. The combined fit using tracks measured in the central tracker and the muon spectrometer results in $p_{T}$ resolution between $1 \%$ and $5 \%$ for $p_{T}$ values up to $1 \mathrm{TeV}$.

The separation between any two objects (jet, lepton, or photon) is required to be

$\Delta R=\sqrt{\Delta \phi^{2}+\Delta \eta^{2}}>0.3$

Black hole signal events are simulated using the partonlevel BlackMax [27] generator (v2.01.03), followed by a partonshowering fragmentation with PYTHIA [28] (v6.420), and a fast parametric simulation of the CMS detector response [29], which has been extensively validated for signal events using detailed detector simulation via GEANT4 [30].

Several parameters govern black hole production and decay in the ADD model in addition to $M_{D}$ and $n$. For each value of $M_{D}$, we consider a range of the minimum black hole masses, $M_{\mathrm{BH}}^{\min }$, between $M_{D}$ and the kinematic limit of the LHC. We assume that no parton-collision energy is lost in gravitational shock waves, i.e. it is all trapped within the event horizon of the forming black hole. We consider both rotating and non-rotating black holes in this analysis, although the description of rotating black holes in the existing MC generators is only approximate. Graviton radiation by the black hole is not considered. For most of the signal samples we assume full Hawking evaporation without a stable non-interacting remnant.

The parameters used in the simulations are listed in Table 1 for a number of characteristic model points. The MSTW2008lo68 [31] parton distribution functions (PDF) were used. In addition we compare the BlackMax results with those of the CHARYBDIS 2 MC 
Table 1

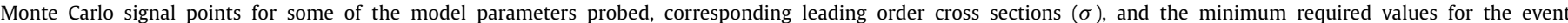

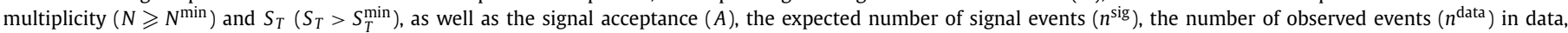
the expected number of background events $\left(n^{\mathrm{bkg}}\right)$, and the observed $\left(\sigma^{95}\right)$ and expected $\left(\sigma_{\text {exp. }}^{95}\right)$ limits on the signal cross section at $95 \%$ confidence level.

\begin{tabular}{|c|c|c|c|c|c|c|c|c|c|c|c|}
\hline$M_{D}(\mathrm{TeV})$ & $M_{\mathrm{BH}}^{\min }(\mathrm{TeV})$ & $n$ & $\sigma(\mathrm{pb})$ & $N^{\min }$ & $S_{T}^{\min }(\mathrm{TeV})$ & $A(\%)$ & $n^{\text {sig }}$ & $n^{\text {data }}$ & $n^{\text {bkg }}$ & $\sigma^{95}(\mathrm{pb})$ & $\sigma_{\text {exp. }}^{95}(\mathrm{pb})$ \\
\hline 1.5 & 2.5 & 6 & 118 & 3 & 1.5 & 90.6 & 3713 & 203 & $241 \pm 45$ & 1.69 & 2.52 \\
\hline 1.5 & 3.0 & 6 & 25.9 & 3 & 1.8 & 91.3 & 823 & 45 & $66.2 \pm 22.2$ & 0.62 & 1.13 \\
\hline 1.5 & 3.5 & 6 & 4.97 & 4 & 2.1 & 88.3 & 153 & 6 & $12.1 \pm 6.3$ & 0.21 & 0.39 \\
\hline 1.5 & 4.0 & 6 & 0.77 & 5 & 2.4 & 84.4 & 22.5 & 0 & $2.01 \pm 1.48$ & 0.11 & 0.18 \\
\hline 1.5 & 4.5 & 6 & 0.09 & 5 & 2.9 & 80.9 & 2.55 & 0 & $0.46_{-0.46}^{+0.54}$ & 0.11 & 0.13 \\
\hline 1.5 & 5.0 & 6 & 0.007 & 5 & 3.4 & 75.2 & 0.19 & 0 & $0.13_{-0.13}^{+0.21}$ & 0.12 & 0.13 \\
\hline 2.0 & 2.5 & 4 & 28.9 & 3 & 1.7 & 81.4 & 817 & 82 & $99.7 \pm 28.1$ & 1.16 & 1.64 \\
\hline 2.0 & 3.0 & 4 & 6.45 & 3 & 2.0 & 83.2 & 186 & 21 & $30.8 \pm 14.0$ & 0.47 & 0.76 \\
\hline 2.0 & 3.5 & 4 & 1.26 & 4 & 2.3 & 77.9 & 34.0 & 3 & $6.12 \pm 4.05$ & 0.20 & 0.31 \\
\hline 2.0 & 4.0 & 4 & 0.20 & 4 & 2.8 & 73.4 & 5.07 & 0 & $1.35_{-1.35}^{+1.45}$ & 0.12 & 0.19 \\
\hline 2.0 & 4.5 & 4 & 0.02 & 5 & 3.2 & 64.4 & 0.53 & 0 & $0.21_{-0.21}^{+0.31}$ & 0.14 & 0.15 \\
\hline 2.0 & 5.0 & 4 & 0.002 & 5 & 3.7 & 59.6 & 0.04 & 0 & $0.06_{-0.06}^{+0.12}$ & 0.15 & 0.15 \\
\hline 3.0 & 3.0 & 2 & 0.59 & 3 & 2.4 & 62.1 & 12.8 & 2 & $7.88 \pm 5.80$ & 0.21 & 0.46 \\
\hline 3.0 & 3.5 & 2 & 0.12 & 3 & 2.8 & 58.9 & 2.41 & 0 & $2.40_{-2.40}^{+2.57}$ & 0.15 & 0.28 \\
\hline 3.0 & 4.0 & 2 & 0.02 & 4 & 3.2 & 47.3 & 0.32 & 0 & $0.46_{-0.46}^{+0.67}$ & 0.19 & 0.23 \\
\hline 3.0 & 4.5 & 2 & 0.002 & 5 & 3.6 & 33.6 & 0.03 & 0 & $0.08_{-0.08}^{+0.15}$ & 0.26 & 0.28 \\
\hline 3.0 & 5.0 & 2 & 0.0002 & 5 & 4.0 & 34.5 & 0.002 & 0 & $0.03_{-0.03}^{+0.07}$ & 0.26 & 0.26 \\
\hline
\end{tabular}

generator (v1.0.3) [32,33]. The two generators yield different values of total cross section, as BlackMax introduces an additional $n$-dependent factor applied on top of the geometrical cross section. The CHARYBDIS cross sections are a factor of $1.36,1.59$, and 1.78 smaller than those from BlackMax for $n=2,4$, and 6 , respectively. In addition, CHARYBDIS has been used to simulate black hole evaporation resulting in a stable non-interacting remnant with mass $M_{D}$ (this model is not implemented in BlackMax). In the generation, we use the Particle Data Group [5] definition of the Planck scale $M_{D}$. (Using another popular choice for $M_{D}$ from Dimopoulos and Landsberg [1] would result in a suppression of the production cross section by a factor of $1.35,5.21$, or 9.29 for $n=2,4$, or 6 , respectively.)

We employ a selection based on total transverse energy to separate black hole candidate events from the backgrounds. The variable $S_{T}$ is defined as a scalar sum of the $E_{T}$ of the $N$ individual objects (jets, electrons, photons, and muons) passing the above selections. Only objects with $E_{T}>50 \mathrm{GeV}$ are included in the calculation of $S_{T}$, in order to suppress the SM backgrounds and to be insensitive to jets from pile-up, while being fully efficient for black hole decays. Further, the missing transverse energy in the event is added to $S_{T}$, if the missing transverse energy value exceeds $50 \mathrm{GeV}$. Note that while $\mathbb{E}_{\mathrm{T}}$ is counted toward $S_{T}$, it is not considered in the determination of $N$.

The main background to black hole signals arises from QCD multijet events. Other backgrounds from direct photon, $W / Z+$ jets, and $t \bar{t}$ production were estimated from MC simulations, using the MADGRAPH [34] leading-order parton-level event generator with CTEQ6L PDF set [35], followed by PYTHIA [28] parton showering and full CMS detector simulation via GEANT4 [30]. These additional backgrounds are negligible at large values of $S_{T}$ and contribute less than $1 \%$ to the total background after the final selection.

The dominant multijet background can only be estimated reliably from data. For QCD events, $S_{T}$ is almost completely determined by the hard $2 \rightarrow 2$ parton scattering process. Further splitting of the jets due to final-state radiation, as well as additional jets due to initial-state radiation - most often nearly collinear with either incoming or outgoing partons - does not change the $S_{T}$ value considerably. Consequently, the shape of the $S_{T}$ distribution is expected to be independent of the event multiplicity $N$, as long as $S_{T}$ is sufficiently above the turn-on region (i.e., much higher than $N \times 50 \mathrm{GeV}$ ). This shape invariance offers a direct way of extracting the expected number of background events in the search for black hole production.

We confirmed the assumption of the $S_{T}$ shape invariance of $N$ up to high multiplicities using MC generators capable of simulating multijet final states from either matrix elements [36] or parton showers [28]. The conjecture that the $S_{T}$ shape is independent of the multiplicity has been also checked with data using the exclusive multiplicities of $N=2$ and $N=3$. Even in the presence of a black hole signal with a mass of a few $\mathrm{TeV}$, the decays of these black holes result in events with half-a-dozen objects in the final state. Hence, the signal contribution to the $N=2$ and $N=3$ data is expected to be small and only seen at large values of $S_{T}$, so these samples still can be used for the background prediction at higher multiplicities. Moreover, since dedicated analyses of the dijet invariant mass spectrum have been conducted $[19,20]$, we know that there are no appreciable contributions from new physics to the dijet final state up to invariant masses of about $1.5 \mathrm{TeV}$, which, for central jets, translates to a similar range of $S_{T}$.

We fit the $S_{T}$ distributions between 600 and $1100 \mathrm{GeV}$, where no black hole signal is expected, for data events with $N=2$ and

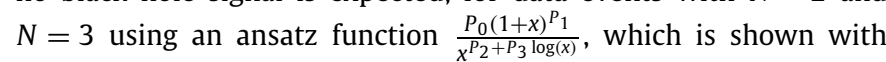
the solid line in Fig. 1. To check the systematic uncertainty of the fit, we use two additional ansatz functions, $\frac{P_{0}}{\left(P_{1}+P_{2} x+x^{2}\right)^{P_{3}}}$ and $\frac{P_{0}}{\left(P_{1}+x\right)^{P_{2}}}$ [19], which are shown as the upper and lower boundaries of the shaded band in Fig. 1. The default choice of the ansatz function was made based on the best-fit to the $S_{T}$ distribution for $N=2$. Additional systematic uncertainty arises from a slight difference between the best-fit shapes for $N=2$ and $N=3$. Nevertheless, the fits for these two exclusive multiplicities agree with each other within the uncertainties, demonstrating that the shape of the $S_{T}$ distribution is independent of the final-state multiplicity.

The $S_{T}$ distributions for data events with multiplicities $N \geqslant 3$, 4, and 5 are shown in Figs. 2a, b, and c, respectively. The solid curves in the figures are the predicted background shapes, found by normalizing the fits of the $N=2 S_{T}$ distribution to the range 

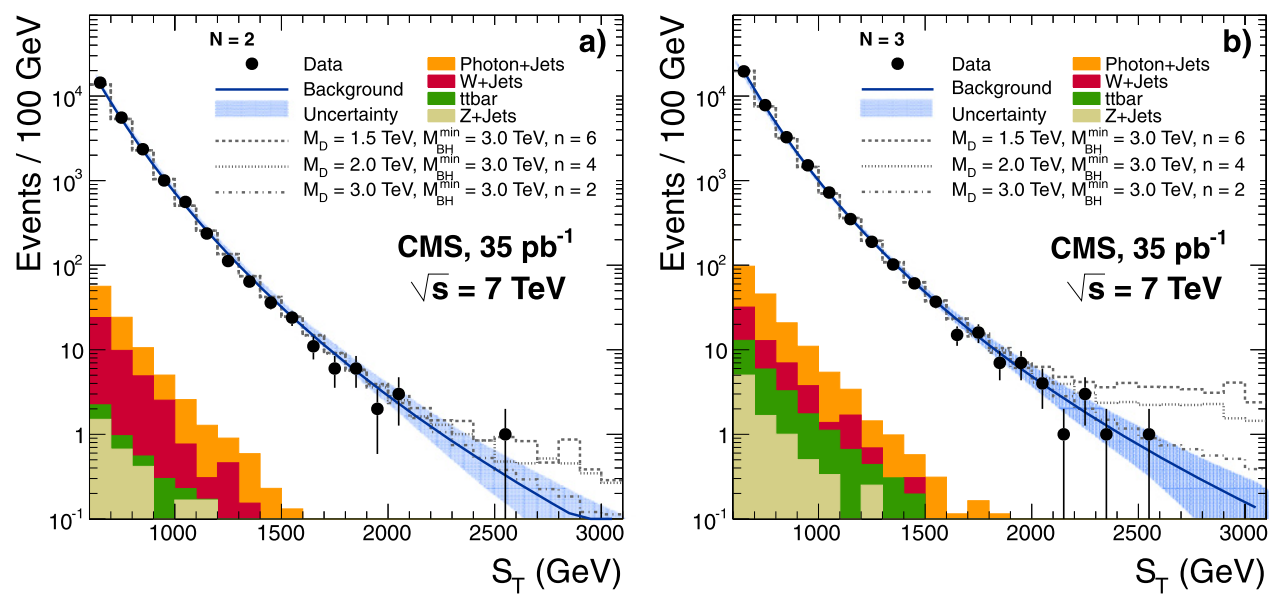

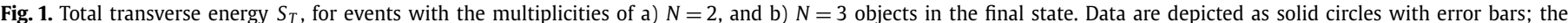

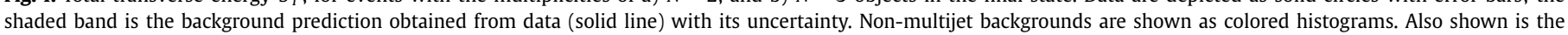
predicted black hole signal for three different parameter sets.
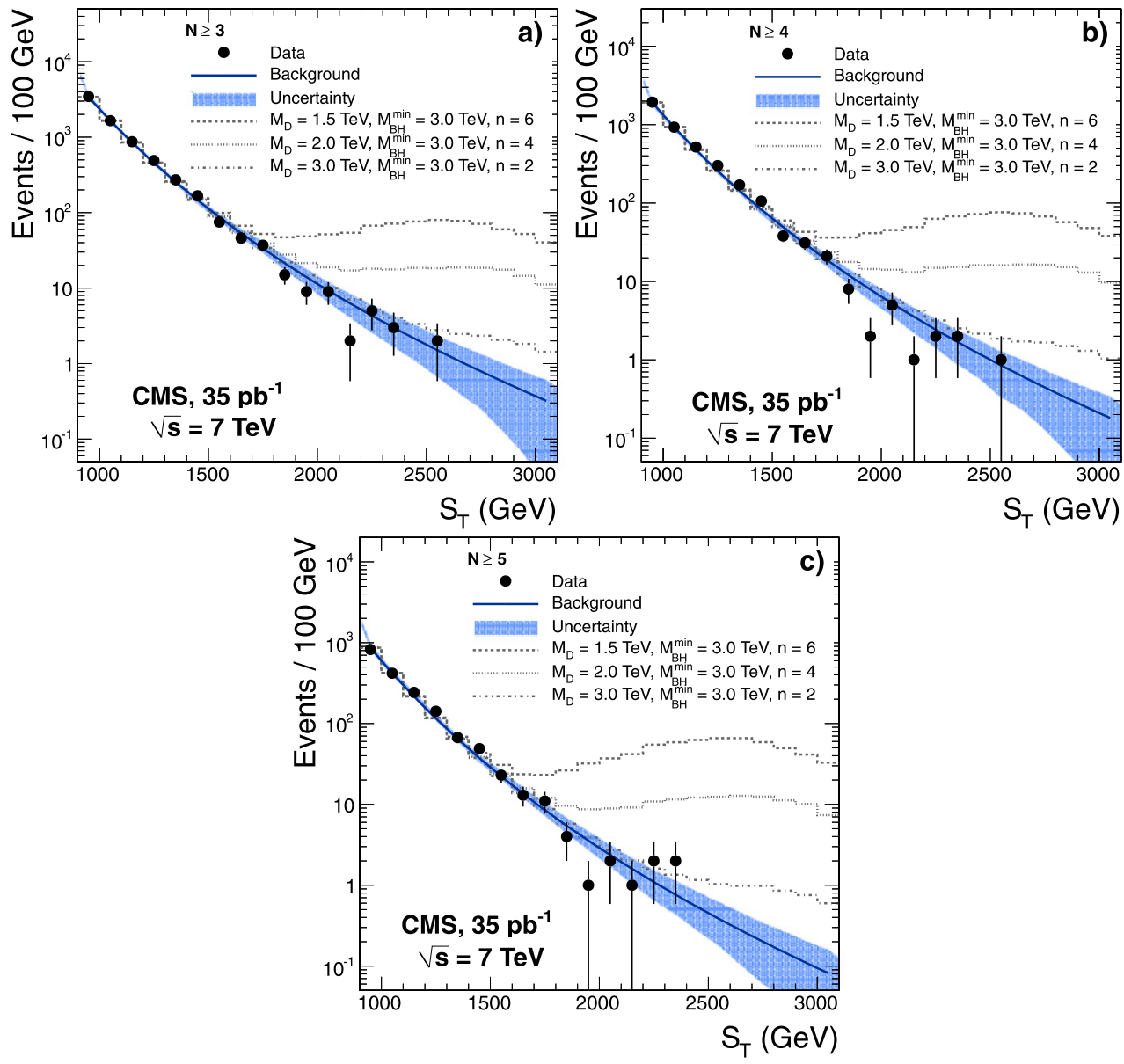

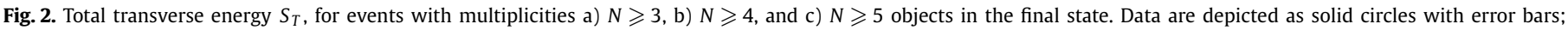
the shaded band is the background prediction (solid line) with its uncertainty. Also shown are black hole signals for three different parameter sets.

$S_{T}=1000-1100 \mathrm{GeV}$, where no black hole signal contribution is expected.

Since no excess is observed above the predicted background, we set limits on the black hole production. We assign a systematic uncertainty on the background estimate of $6 \%$ to $125 \%$ for the $S_{T}$ range used in this search. This uncertainty comes from the normalization uncertainty (4-12\%, dominated by the statistics in the normalization region) added in quadrature to the uncertainties arising from using various ansatz fit functions and the difference between the shapes obtained from the $N=2$ and $N=3$ samples. The integrated luminosity is measured with an uncertainty of $11 \%$ [22]. The uncertainty on the signal yield is dominated by the jet-energy-scale uncertainty of $\approx 5 \%$ [24] which translates into a $5 \%$ uncertainty on the signal. An additional $2 \%$ uncertainty on 


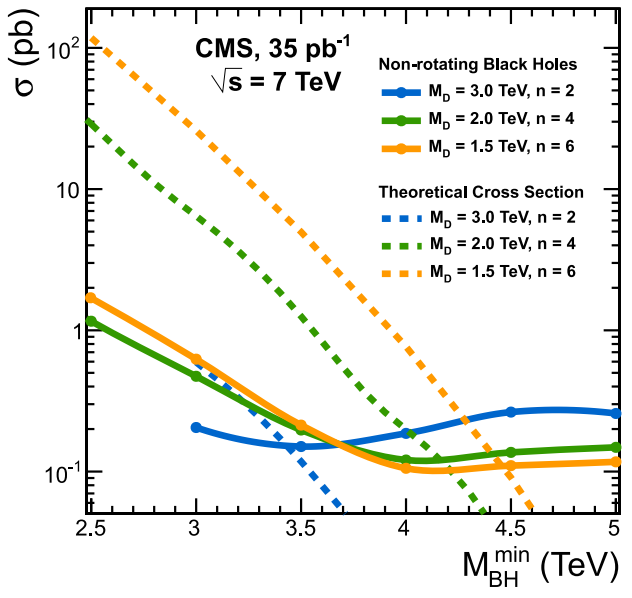

Fig. 3. The $95 \%$ confidence level upper limits on the black hole production cross section (solid lines) and three theoretical predictions for the cross section (dashed lines), as a function of the black hole mass.

the signal acceptance comes from the variation of PDFs within the CTEQ6 error set [35]. The particle identification efficiency does not affect the signal distribution, since an electron failing the identification requirements would be classified either as a photon or a jet; a photon failing the selection would become a jet; a rejected muon would contribute to the $\mathbb{E}_{\mathrm{T}}$. In any case the total value of $S_{T}$ is not affected.

We set limits on black hole production with the optimized $S_{T}$ and $N$ selections by counting events with $S_{T}>S_{T}^{\min }$ and $N>N^{\min }$. We optimized the signal $(S)$ significance in the presence of background $(B)$ using the ratio $S / \sqrt{S+B}$ for each set. The optimum choice of parameters is listed in Table 1 , as well as the predicted number of background events, the expected number of signal events, and the observed number of events in data. Note that the background uncertainty, dominated by the choice of the fitting function, is highly correlated for various working points listed in Table 1 and also bin-to-bin for the $S_{T}$ distributions shown in Figs. 1 and 2 .

We set upper limits on the black hole production cross section using the Bayesian method with flat signal prior and log-normal prior for integration over the nuisance parameters (background, signal acceptance, luminosity) [5,37]. These upper limits at the $95 \%$ confidence level (CL) are shown in Fig. 3, as a function of $M_{\mathrm{BH}}^{\mathrm{min}}$. For the three model parameter sets shown in the figure, the observed (expected) lower limits on the black hole mass are 3.5, 4.2 and $4.5 \mathrm{TeV}(3.2,4.0$, and $4.5 \mathrm{TeV})$, respectively.

Translating these upper limits into lower limits on the parameters of the ADD model, we can exclude the production of black holes with minimum mass of 3.5-4.5 TeV for values of the multidimensional Planck scale up to $3.5 \mathrm{TeV}$ at 95\% CL. These limits, shown in Fig. 4, do not exhibit significant dependence on the details of the production and evaporation within the set of models we studied. These are the first limits of a dedicated search for black hole production at a particle accelerator.

We point out that the semi-classical approximation used in this search is valid only for the lowest values of the $M_{D}$, for which the limits on the minimum black hole mass exceed $M_{D}$ by a factor of a few. For higher values of $M_{D}$ the limits become comparable with $M_{D}$, which implies that the approximation is no longer valid and that the BH production cross section may be modified significantly. Nevertheless, due to the exponentially falling nature of production cross section with the black hole mass, even large changes in the cross section translate only in moderate changes in the minimum black hole mass limit, as evident from Fig. 3.

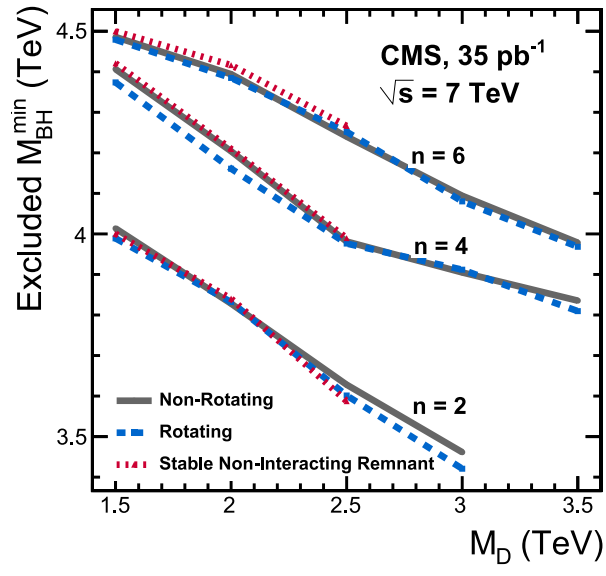

Fig. 4. The 95\% confidence level limits on the black hole mass as a function of the multidimensional Planck scale $M_{D}$ for several benchmark scenarios. The area below each curve is excluded by this search.

Finally, we produce model-independent upper limits on the cross section times the acceptance for new physics production in high- $S_{T}$ inclusive final states for $N \geqslant 3,4$, and 5. Fig. 5 shows 95\% CL upper limits from a counting experiment for $S_{T}>S_{T}^{\min }$ as a function of $S_{T}^{\mathrm{min}}$, which can be used to test models of new physics that result in these final states. A few examples of such models are production of high-mass $t \bar{t}$ resonances [38] in the sixjet and lepton + jet final states, $R$-parity violating gluino decay into three jets, resulting in the six-jet final state $[39,40]$, and a class of models with strong dynamics, with a strongly produced resonance decaying into a pair of resonances further decaying into two jets each, resulting in the four-jet final state [41]. In addition, these limits can be used to constrain black hole production for additional regions of the parameter space of the model, as well as set limits on the existence of string balls [42], which are quantum precursors of black holes predicted in certain string models. We have checked that for the black hole model parameters we probed with the dedicated optimized analysis, the sensitivity of the search in terms of the excluded black hole mass range exceeds that from the model-independent cross section limits by as little as $5-8 \%$. Thus, model-independent limits can be used efficiently to constrain the allowed parameter space in an even broader variety of black hole models than we covered in this Letter.

To conclude, we have performed the first dedicated search for microscopic black holes at a particle accelerator and set limits on their production in the model with large extra dimensions in space using simple semi-classical approximation of the black hole production and decay $[1,2]$. The lower limits on the black hole mass at $95 \% \mathrm{CL}$ range from 3.5 to $4.5 \mathrm{TeV}$ for values of the Planck scale up to $3 \mathrm{TeV}$. Additionally, we have produced model-independent limits on the production of energetic, high-multiplicity final states, which can be used to constrain a variety of models of new physics.

\section{Acknowledgements}

We wish to congratulate our colleagues in the CERN accelerator departments for the excellent performance of the LHC machine. We thank the technical and administrative staff at CERN and other CMS institutes, and acknowledge support from: FMSR (Austria); FNRS and FWO (Belgium); CNPq, CAPES, FAPERJ, and FAPESP (Brazil); MES (Bulgaria); CERN; CAS, MoST, and NSFC (China); COLCIENCIAS (Colombia); MSES (Croatia); RPF (Cyprus); Academy of Sciences and NICPB (Estonia); Academy of Finland, ME, and HIP (Finland); CEA and CNRS/IN2P3 (France); BMBF, DFG, and HGF 

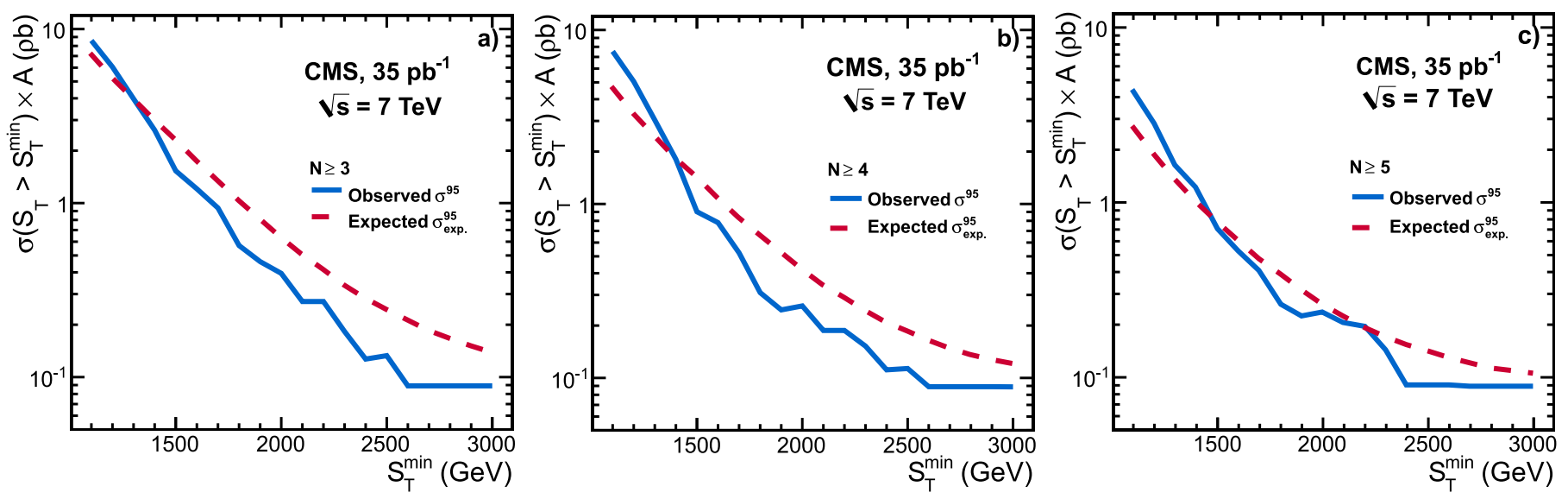

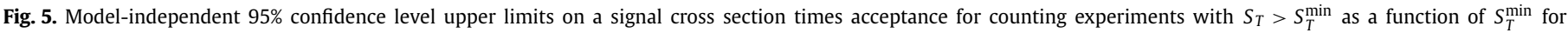
(a) $N \geqslant 3$, (b) $N \geqslant 4$, and (c) $N \geqslant 5$. The solid (dashed) lines correspond to an observed (expected) limit for nominal signal acceptance uncertainty of $5 \%$.

(Germany); GSRT (Greece); OTKA and NKTH (Hungary); DAE and DST (India); IPM (Iran); SFI (Ireland); INFN (Italy); NRF and WCU (Korea); LAS (Lithuania); CINVESTAV, CONACYT, SEP, and UASLPFAI (Mexico); PAEC (Pakistan); SCSR (Poland); FCT (Portugal); JINR (Armenia, Belarus, Georgia, Ukraine, Uzbekistan); MST and MAE (Russia); MSTD (Serbia); MICINN and CPAN (Spain); Swiss Funding Agencies (Switzerland); NSC (Taipei); TUBITAK and TAEK (Turkey); STFC (United Kingdom); DOE and NSF (USA).

\section{Open access}

This article is published Open Access at sciencedirect.com. It is distributed under the terms of the Creative Commons Attribution License 3.0, which permits unrestricted use, distribution, and reproduction in any medium, provided the original authors and source are credited.

\section{References}

[1] S. Dimopoulos, G. Landsberg, Phys. Rev. Lett. 87 (2001) 161602, doi:10.1103/ PhysRevLett.87.161602, arXiv:hep-ph/0106295.

[2] S. Giddings, S. Thomas, Phys. Rev. D 65 (2002) 056010, doi:10.1103/ PhysRevD.65.056010, arXiv:hep-ph/0106219.

[3] N. Arkani-Hamed, S. Dimopoulos, G. Dvali, Phys. Lett. B 429 (1998) 263, doi:10.1016/S0370-2693(98)00466-3, arXiv:hep-ph/9803315.

[4] N. Arkani-Hamed, S. Dimopoulos, G. Dvali, Phys. Rev. D 59 (1999) 086004, doi:10.1103/PhysRevD.59.086004, arXiv:hep-ph/9807344.

[5] K. Nakamura, et al., J. Phys. G 37 (2010) 075021, doi:10.1088/0954-3899/37/ 7A/075021.

[6] F. Tangherlini, Nuovo Cimento 27 (1963) 636, doi:10.1007/BF02784569.

[7] R. Myers, M. Perry, Ann. Phys. 172 (1986) 304, doi:10.1016/00034916(86)90186-7.

[8] P. Argyres, S. Dimopoulos, J. March-Russell, Phys. Lett. B 441 (1998) 96, doi:10.1016/S0370-2693(98)01184-8, arXiv:hep-th/9808138.

[9] H. Yoshino, Y. Nambu, Phys. Rev. D 67 (2003) 024009, doi:10.1103/ PhysRevD.67.024009, arXiv:gr-qc/0209003.

[10] P. Kanti, Int. J. Mod. Phys. A 19 (2004) 4899, doi:10.1142/S0217751X04018324, arXiv:hep-ph/0402168.

[11] G. Landsberg, J. Phys. G 32 (2006) R337, doi:10.1088/0954-3899/32/9/R02, arXiv:hep-ph/0607297.

[12] S. Hawking, Commun. Math. Phys. 43 (1975) 199, doi:10.1007/BF02345020.

[13] R. Emparan, G. Horowitz, R. Myers, Phys. Rev. Lett. 85 (2000) 499, doi:10.1103/ PhysRevLett.85.499, arXiv:hep-th/0003118.

[14] P. Kanti, H. Kodama, R.A. Konoplya, N. Pappas, A. Zhidenko, Phys. Rev. D 80 (2009) 084016, doi:10.1103/PhysRevD.80.084016, arXiv:0906.3845.

[15] B. Koch, M. Bleicher, S. Hossenfelder, JHEP 0510 (2005) 053, doi:10.1088/11266708/2005/10/053, arXiv:hep-ph/0507138.

[16] P. Meade, L. Randall, JHEP 0805 (2008) 003, doi:10.1088/1126-6708/2008/ 05/003, arXiv:0708.3017.

[17] X. Calmet, W. Gong, S. Hsu, Phys. Lett. B 668 (2008) 20, doi:10.1016/ j.physletb.2008.08.011, arXiv:0806.4605.
[18] D. Gingrich, J. Phys. G 37 (2010) 105108, doi:10.1088/0954-3899/37/10/105008, arXiv:0912.0826.

[19] CMS Collaboration, Phys. Rev. Lett. 105 (2010) 211801, doi:10.1103/ PhysRevLett.105.211801, arXiv:1010.0203.

[20] CMS Collaboration, Phys. Rev. Lett. 105 (2010) 262001, doi:10.1103/ PhysRevLett.105.262001, arXiv:1010.4439.

[21] CMS Collaboration, JINST 3 (2008) S08004, doi:10.1088/1748-0221/3/08/ S08004.

[22] CMS Collaboration, Measurement of CMS luminosity, CMS Physics Analysis Summary CMS-PAS-EWK-10-004.

[23] M. Cacciari, G. Salam, G. Soyez, JHEP 0804 (2008) 063, doi:10.1088/11266708/2008/04/063, arXiv:0802.1189.

[24] CMS Collaboration, Jet energy corrections determination at $7 \mathrm{TeV}, \mathrm{CMS}$ Physics Analysis Summary CMS-PAS-JME-10-010, URL http://cdsweb.cern.ch/record/ 1308178.

[25] CMS Collaboration, Missing transverse energy performance in minimum-bias and jet events from proton-proton collisions at $\sqrt{s}=7 \mathrm{TeV}$, CMS Physics Analysis Summary CMS-PAS-JME-10-004, URL http://cdsweb.cern.ch/record/ 1279142.

[26] P. Adzic, et al., JINST 2 (2007) P04004, doi:10.1088/1748-0221/2/04/ P04004.

[27] D.-C. Dai, G. Starkman, D. Stojkovic, C. Issever, E. Rizvi, J. Tseng, Phys. Rev. D 77 (2008) 076007, doi:10.1103/PhysRevD.77.076007, arXiv:0711.3012.

[28] T. Sjöstrand, S. Mrenna, P. Skands, JHEP 0605 (2006) 026, doi:10.1088/11266708/2006/05/026, arXiv:hep-ph/0603175.

[29] D. Orbaker, J. Phys. Conf. Ser. 219 (2010) 032053, doi:10.1088/1742-6596/ 219/3/032053.

[30] S. Agostinelli, et al., Nucl. Instrum. Methods A 506 (2003) 250, doi:10.1016/ S0168-9002(03)01368-8.

[31] A. Martin, W. Stirling, R. Thorne, G. Watt, Eur. Phys. J. C 70 (2010) 51, doi:10.1140/epjc/s10052-010-1462-8, arXiv:1007.2624.

[32] C. Harris, P. Richardson, B. Webber, JHEP 0308 (2003) 033, doi:10.1088/11266708/2003/08/033, arXiv:hep-ph/0307305.

[33] J. Frost, et al., JHEP 0910 (2009) 014, doi:10.1088/1126-6708/2009/10/014, arXiv:0904.0979, URL http://projects.hepforge.org/charybdis2/.

[34] J. Alwall, et al., JHEP 0709 (2007) 028, doi:10.1088/1126-6708/2007/09/028, arXiv:0706.2334.

[35] P. Nadolsky, et al., Phys. Rev. D 78 (2008) 013004, doi:10.1103/PhysRevD. 78.013004, arXiv:0802.0007.

[36] M. Mangano, M. Moretti, F. Piccinini, R. Pittau, A. Polosa, JHEP 0307 (2003) 001, doi:10.1088/1126-6708/2003/07/001, arXiv:hep-ph/0206293.

[37] I. Bertram, G. Landsberg, J. Linnemann, R. Partridge, M. Paterno, H. Prosper, A recipe for the construction of confidence limits, Tech. rep., FERMILAB-TM2104, 2000.

[38] K. Agashe, A. Belyaev, T. Krupovnickas, G. Perez, J. Virzi, Phys. Rev. D 77 (2008) 015003, doi:10.1103/PhysRevD.77.015003, arXiv:hep-ph/0612015.

[39] R. Chivukula, M. Golden, E. Simmons, Phys. Lett. B 257 (1991) 403, doi:10.1016/0370-2693(91)91915-I.

[40] R. Chivukula, M. Golden, E. Simmons, Nucl. Phys. B 363 (1991) 83, doi:10.1016/0550-3213(91)90235-P.

[41] B. Dobrescu, K. Kong, R. Mahbubani, Phys. Lett. B 670 (2008) 119, doi:10.1016/j.physletb.2008.10.048, arXiv:0709.2378v3 [hep-ph].

[42] S. Dimopoulos, R. Emparan, Phys. Lett. B 526 (2002) 393, doi:10.1016/S03702693(01)01525-8, arXiv:hep-ph/0108060. 


\section{CMS Collaboration}

V. Khachatryan, A.M. Sirunyan, A. Tumasyan

Yerevan Physics Institute, Yerevan, Armenia

W. Adam, T. Bergauer, M. Dragicevic, J. Erö, C. Fabjan, M. Friedl, R. Frühwirth, V.M. Ghete, J. Hammer ${ }^{1}$, S. Hänsel, C. Hartl, M. Hoch, N. Hörmann, J. Hrubec, M. Jeitler, G. Kasieczka, W. Kiesenhofer, M. Krammer, D. Liko, I. Mikulec, M. Pernicka, H. Rohringer, R. Schöfbeck, J. Strauss, A. Taurok, F. Teischinger, W. Waltenberger, G. Walzel, E. Widl, C.-E. Wulz

Institut für Hochenergiephysik der OeAW, Wien, Austria

V. Mossolov, N. Shumeiko, J. Suarez Gonzalez

National Centre for Particle and High Energy Physics, Minsk, Belarus

L. Benucci, K. Cerny, E.A. De Wolf, X. Janssen, T. Maes, L. Mucibello, S. Ochesanu, B. Roland, R. Rougny, M. Selvaggi, H. Van Haevermaet, P. Van Mechelen, N. Van Remortel

Universiteit Antwerpen, Antwerpen, Belgium

V. Adler, S. Beauceron, F. Blekman, S. Blyweert, J. D’Hondt, O. Devroede, R. Gonzalez Suarez,

A. Kalogeropoulos, J. Maes, M. Maes, S. Tavernier, W. Van Doninck, P. Van Mulders, G.P. Van Onsem, I. Villella

Vrije Universiteit Brussel, Brussel, Belgium

O. Charaf, B. Clerbaux, G. De Lentdecker, V. Dero, A.P.R. Gay, G.H. Hammad, T. Hreus, P.E. Marage, L. Thomas, C. Vander Velde, P. Vanlaer, J. Wickens

Université Libre de Bruxelles, Bruxelles, Belgium

S. Costantini, M. Grunewald, B. Klein, A. Marinov, J. Mccartin, D. Ryckbosch, F. Thyssen, M. Tytgat, L. Vanelderen, P. Verwilligen, S. Walsh, N. Zaganidis

Ghent University, Ghent, Belgium

S. Basegmez, G. Bruno, J. Caudron, L. Ceard, J. De Favereau De Jeneret, C. Delaere, P. Demin, D. Favart, A. Giammanco, G. Grégoire, J. Hollar, V. Lemaitre, J. Liao, O. Militaru, S. Ovyn, D. Pagano, A. Pin, K. Piotrzkowski, L. Quertenmont, N. Schul

Université Catholique de Louvain, Louvain-la-Neuve, Belgium

N. Beliy, T. Caebergs, E. Daubie

Université de Mons, Mons, Belgium

G.A. Alves, D. De Jesus Damiao, M.E. Pol, M.H.G. Souza

Centro Brasileiro de Pesquisas Fisicas, Rio de Janeiro, Brazil

W. Carvalho, E.M. Da Costa, C. De Oliveira Martins, S. Fonseca De Souza, L. Mundim, H. Nogima, V. Oguri, W.L. Prado Da Silva, A. Santoro, S.M. Silva Do Amaral, A. Sznajder,

F. Torres Da Silva De Araujo

Universidade do Estado do Rio de Janeiro, Rio de Janeiro, Brazil

F.A. Dias, M.A.F. Dias, T.R. Fernandez Perez Tomei, E.M. Gregores ${ }^{2}$, F. Marinho, S.F. Novaes, Sandra S. Padula 
N. Darmenov ${ }^{1}$, L. Dimitrov, V. Genchev ${ }^{1}$, P. Iaydjiev ${ }^{1}$, S. Piperov, M. Rodozov, S. Stoykova, G. Sultanov, V. Tcholakov, R. Trayanov, I. Vankov

Institute for Nuclear Research and Nuclear Energy, Sofia, Bulgaria

M. Dyulendarova, R. Hadjiiska, V. Kozhuharov, L. Litov, E. Marinova, M. Mateev, B. Pavlov, P. Petkov

University of Sofia, Sofia, Bulgaria

J.G. Bian, G.M. Chen, H.S. Chen, C.H. Jiang, D. Liang, S. Liang, J. Wang, J. Wang, X. Wang, Z. Wang, M. Xu, M. Yang, J. Zang, Z. Zhang

Institute of High Energy Physics, Beijing, China

Y. Ban, S. Guo, W. Li, Y. Mao, S.J. Qian, H. Teng, L. Zhang, B. Zhu

State Key Lab. of Nucl. Phys. and Tech., Peking University, Beijing, China

A. Cabrera, B. Gomez Moreno, A.A. Ocampo Rios, A.F. Osorio Oliveros, J.C. Sanabria

Universidad de Los Andes, Bogota, Colombia

N. Godinovic, D. Lelas, K. Lelas, R. Plestina ${ }^{3}$, D. Polic, I. Puljak

Technical University of Split, Split, Croatia

Z. Antunovic, M. Dzelalija

University of Split, Split, Croatia

V. Brigljevic, S. Duric, K. Kadija, S. Morovic

Institute Rudjer Boskovic, Zagreb, Croatia

A. Attikis, M. Galanti, J. Mousa, C. Nicolaou, F. Ptochos, P.A. Razis, H. Rykaczewski University of Cyprus, Nicosia, Cyprus

Y. Assran ${ }^{4}$, A. Awad

Academy of Scientific Research and Technology of the Arab Republic of Egypt, Egyptian Network of High Energy Physics, Cairo, Egypt

A. Hektor, M. Kadastik, K. Kannike, M. Müntel, M. Raidal, L. Rebane

National Institute of Chemical Physics and Biophysics, Tallinn, Estonia

V. Azzolini, P. Eerola

Department of Physics, University of Helsinki, Helsinki, Finland

S. Czellar, J. Härkönen, A. Heikkinen, V. Karimäki, R. Kinnunen, J. Klem, M.J. Kortelainen, T. Lampén, K. Lassila-Perini, S. Lehti, T. Lindén, P. Luukka, T. Mäenpää, E. Tuominen, J. Tuominiemi, E. Tuovinen, D. Ungaro, L. Wendland

Helsinki Institute of Physics, Helsinki, Finland

K. Banzuzi, A. Korpela, T. Tuuva

Lappeenranta University of Technology, Lappeenranta, Finland

D. Sillou

Laboratoire d'Annecy-le-Vieux de Physique des Particules, IN2P3-CNRS, Annecy-le-Vieux, France 
M. Besancon, S. Choudhury, M. Dejardin, D. Denegri, B. Fabbro, J.L. Faure, F. Ferri, S. Ganjour, F.X. Gentit, A. Givernaud, P. Gras, G. Hamel de Monchenault, P. Jarry, E. Locci, J. Malcles, M. Marionneau, L. Millischer, J. Rander, A. Rosowsky, I. Shreyber, M. Titov, P. Verrecchia

DSM/IRFU, CEA/Saclay, Gif-sur-Yvette, France

S. Baffioni, F. Beaudette, L. Bianchini, M. Bluj ${ }^{5}$, C. Broutin, P. Busson, C. Charlot, T. Dahms, L. Dobrzynski, R. Granier de Cassagnac, M. Haguenauer, P. Miné, C. Mironov, C. Ochando, P. Paganini, D. Sabes, R. Salerno, Y. Sirois, C. Thiebaux, B. Wyslouch ${ }^{6}$, A. Zabi

Laboratoire Leprince-Ringuet, Ecole Polytechnique, IN2P3-CNRS, Palaiseau, France

J.-L. Agram ${ }^{7}$, J. Andrea, A. Besson, D. Bloch, D. Bodin, J.-M. Brom, M. Cardaci, E.C. Chabert, C. Collard, E. Conte ${ }^{7}$, F. Drouhin ${ }^{7}$, C. Ferro, J.-C. Fontaine ${ }^{7}$, D. Gelé, U. Goerlach, S. Greder, P. Juillot, M. Karim ${ }^{7}$, A.-C. Le Bihan, Y. Mikami, P. Van Hove

Institut Pluridisciplinaire Hubert Curien, Université de Strasbourg, Université de Haute Alsace Mulhouse, CNRS/IN2P3, Strasbourg, France

\section{F. Fassi, D. Mercier}

Centre de Calcul de l'Institut National de Physique Nucleaire et de Physique des Particules (IN2P3), Villeurbanne, France

C. Baty, N. Beaupere, M. Bedjidian, O. Bondu, G. Boudoul, D. Boumediene, H. Brun, N. Chanon, R. Chierici, D. Contardo, P. Depasse, H. El Mamouni, A. Falkiewicz, J. Fay, S. Gascon, B. Ille, T. Kurca, T. Le Grand, M. Lethuillier, L. Mirabito, S. Perries, V. Sordini, S. Tosi, Y. Tschudi, P. Verdier, H. Xiao

Université de Lyon, Université Claude Bernard Lyon 1, CNRS-IN2P3, Institut de Physique Nucléaire de Lyon, Villeurbanne, France

\section{Roinishvili}

E. Andronikashvili Institute of Physics, Academy of Science, Tbilisi, Georgia

G. Anagnostou, M. Edelhoff, L. Feld, N. Heracleous, O. Hindrichs, R. Jussen, K. Klein, J. Merz, N. Mohr, A. Ostapchuk, A. Perieanu, F. Raupach, J. Sammet, S. Schael, D. Sprenger, H. Weber, M. Weber, B. Wittmer RWTH Aachen University, I. Physikalisches Institut, Aachen, Germany

M. Ata, W. Bender, M. Erdmann, J. Frangenheim, T. Hebbeker, A. Hinzmann, K. Hoepfner, C. Hof, T. Klimkovich, D. Klingebiel, P. Kreuzer, D. Lanske ${ }^{\dagger}$, C. Magass, G. Masetti, M. Merschmeyer, A. Meyer, P. Papacz, H. Pieta, H. Reithler, S.A. Schmitz, L. Sonnenschein, J. Steggemann, D. Teyssier

RWTH Aachen University, III. Physikalisches Institut A, Aachen, Germany

M. Bontenackels, M. Davids, M. Duda, G. Flügge, H. Geenen, M. Giffels, W. Haj Ahmad, D. Heydhausen, T. Kress, Y. Kuessel, A. Linn, A. Nowack, L. Perchalla, O. Pooth, J. Rennefeld, P. Sauerland, A. Stahl, M. Thomas, D. Tornier, M.H. Zoeller

RWTH Aachen University, III. Physikalisches Institut B, Aachen, Germany

M. Aldaya Martin, W. Behrenhoff, U. Behrens, M. Bergholz ${ }^{8}$, K. Borras, A. Cakir, A. Campbell, E. Castro, D. Dammann, G. Eckerlin, D. Eckstein, A. Flossdorf, G. Flucke, A. Geiser, I. Glushkov, J. Hauk, H. Jung, M. Kasemann, I. Katkov, P. Katsas, C. Kleinwort, H. Kluge, A. Knutsson, D. Krücker, E. Kuznetsova, W. Lange, W. Lohmann ${ }^{8}$, R. Mankel, M. Marienfeld, I.-A. Melzer-Pellmann, A.B. Meyer, J. Mnich, A. Mussgiller, J. Olzem, A. Parenti, A. Raspereza, A. Raval, R. Schmidt ${ }^{8}$, T. Schoerner-Sadenius, N. Sen, M. Stein, J. Tomaszewska, D. Volyanskyy, R. Walsh, C. Wissing

Deutsches Elektronen-Synchrotron, Hamburg, Germany

C. Autermann, S. Bobrovskyi, J. Draeger, H. Enderle, U. Gebbert, K. Kaschube, G. Kaussen, R. Klanner, J. Lange, B. Mura, S. Naumann-Emme, F. Nowak, N. Pietsch, C. Sander, H. Schettler, 
P. Schleper, M. Schröder, T. Schum, J. Schwandt, A.K. Srivastava, H. Stadie, G. Steinbrück, J. Thomsen, R. Wolf

University of Hamburg, Hamburg, Germany

C. Barth, J. Bauer, V. Buege, T. Chwalek, W. De Boer, A. Dierlamm, G. Dirkes, M. Feindt, J. Gruschke,

C. Hackstein, F. Hartmann, S.M. Heindl, M. Heinrich, H. Held, K.H. Hoffmann, S. Honc, T. Kuhr,

D. Martschei, S. Mueller, Th. Müller, M. Niegel, O. Oberst, A. Oehler, J. Ott, T. Peiffer, D. Piparo, G. Quast,

K. Rabbertz, F. Ratnikov, M. Renz, C. Saout, A. Scheurer, P. Schieferdecker, F.-P. Schilling, G. Schott, H.J. Simonis, F.M. Stober, D. Troendle, J. Wagner-Kuhr, M. Zeise, V. Zhukov ${ }^{9}$, E.B. Ziebarth

Institut für Experimentelle Kernphysik, Karlsruhe, Germany

G. Daskalakis, T. Geralis, S. Kesisoglou, A. Kyriakis, D. Loukas, I. Manolakos, A. Markou, C. Markou, C. Mavrommatis, E. Ntomari, E. Petrakou

Institute of Nuclear Physics “Demokritos”, Aghia Paraskevi, Greece

L. Gouskos, T.J. Mertzimekis, A. Panagiotou ${ }^{1}$

University of Athens, Athens, Greece

I. Evangelou, C. Foudas, P. Kokkas, N. Manthos, I. Papadopoulos, V. Patras, F.A. Triantis

University of Ioánnina, Ioánnina, Greece

A. Aranyi, G. Bencze, L. Boldizsar, G. Debreczeni, C. Hajdu ${ }^{1}$, D. Horvath ${ }^{10}$, A. Kapusi, K. Krajczar ${ }^{11}$, A. Laszlo, F. Sikler, G. Vesztergombi ${ }^{11}$

KFKI Research Institute for Particle and Nuclear Physics, Budapest, Hungary

N. Beni, J. Molnar, J. Palinkas, Z. Szillasi, V. Veszpremi

Institute of Nuclear Research ATOMKI, Debrecen, Hungary

P. Raics, Z.L. Trocsanyi, B. Ujvari

University of Debrecen, Debrecen, Hungary

S. Bansal, S.B. Beri, V. Bhatnagar, N. Dhingra, M. Jindal, M. Kaur, J.M. Kohli, M.Z. Mehta, N. Nishu, L.K. Saini, A. Sharma, A.P. Singh, J.B. Singh, S.P. Singh

Panjab University, Chandigarh, India

S. Ahuja, S. Bhattacharya, B.C. Choudhary, P. Gupta, S. Jain, S. Jain, A. Kumar, R.K. Shivpuri

University of Delhi, Delhi, India

R.K. Choudhury, D. Dutta, S. Kailas, S.K. Kataria, A.K. Mohanty ${ }^{1}$, L.M. Pant, P. Shukla

Bhabha Atomic Research Centre, Mumbai, India

T. Aziz, M. Guchait ${ }^{12}$, A. Gurtu, M. Maity ${ }^{13}$, D. Majumder, G. Majumder, K. Mazumdar, G.B. Mohanty, A. Saha, K. Sudhakar, N. Wickramage

Tata Institute of Fundamental Research - EHEP, Mumbai, India

S. Banerjee, S. Dugad, N.K. Mondal

Tata Institute of Fundamental Research - HECR, Mumbai, India

H. Arfaei, H. Bakhshiansohi, S.M. Etesami, A. Fahim, M. Hashemi, A. Jafari, M. Khakzad, A. Mohammadi, M. Mohammadi Najafabadi, S. Paktinat Mehdiabadi, B. Safarzadeh, M. Zeinali 
M. Abbrescia ${ }^{a, b}$, L. Barbone ${ }^{a, b}$, C. Calabria ${ }^{a, b}$, A. Colaleo $^{a}$, D. Creanza ${ }^{a, c}$, N. De Filippis ${ }^{a, c}$, M. De Palma ${ }^{a, b}, A$. Dimitrov ${ }^{a}$, L. Fiore ${ }^{a}$, G. Iaselli ${ }^{a, c}$, L. Lusito $^{a, b}, 1$, G. Maggi $^{a, c}$, M. Maggi $^{a}$, N. Manna ${ }^{a, b}$, B. Marangelli a,b, S. My a,c, S. Nuzzo ${ }^{a, b}$, N. Pacifico $^{a, b}$, G.A. Pierro a, A. Pompili ${ }^{a, b}$, G. Pugliese ${ }^{a, c}$, F. Romano ${ }^{a, c}$, G. Roselli ${ }^{a, b}$, G. Selvaggi ${ }^{a, b}$, L. Silvestris ${ }^{a}$, R. Trentadue $^{a}$, S. Tupputi ${ }^{\mathrm{a}, \mathrm{b}}, \mathrm{G}$. Zito $^{\mathrm{a}}$

a INFN Sezione di Bari, Bari, Italy

b Università di Bari, Bari, Italy

c Politecnico di Bari, Bari, Italy

G. Abbiendi a , A.C. Benvenuti ${ }^{\text {a }}$, D. Bonacorsi ${ }^{a}$, S. Braibant-Giacomelli ${ }^{\text {a,b }}$, L. Brigliadori ${ }^{\text {a }}$, P. Capiluppi ${ }^{\text {a,b }}$, A. Castro $^{\text {a,b }}$, F.R. Cavallo ${ }^{\text {a }}$, M. Cuffiani ${ }^{\text {a,b }}$, G.M. Dallavalle ${ }^{a}$, F. Fabbri ${ }^{a}$, A. Fanfani ${ }^{\text {a,b }}$, D. Fasanella ${ }^{\text {a }}$, P. Giacomelli a , M. Giunta ${ }^{\text {a }}$, S. Marcellini ${ }^{\text {a }}$, M. Meneghelli ${ }^{a}{ }^{b}$, A. Montanari ${ }^{\text {a }}$, F.L. Navarria ${ }^{\text {a,b }}$, F. Odorici ${ }^{\text {a }}$, A. Perrotta ${ }^{\text {a }}$, F. Primavera ${ }^{\text {a }}$, A.M. Rossi ${ }^{\text {a,b }}$, T. Rovelli ${ }^{a, b}$, G. Siroli $^{\text {a,b }}{ }^{\text {, R. Travaglini }}{ }^{\text {a,b }}$

a INFN Sezione di Bologna, Bologna, Italy

b Università di Bologna, Bologna, Italy

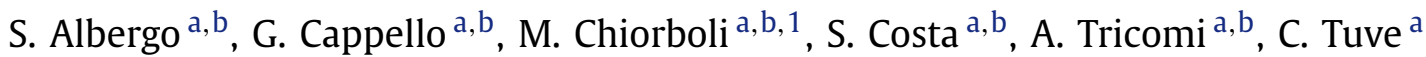

a INFN Sezione di Catania, Catania, Italy

b Università di Catania, Catania, Italy

G. Barbagli ${ }^{a}$, V. Ciulli ${ }^{a, b}$, C. Civinini ${ }^{a}$, R. D’Alessandro ${ }^{a, b}$, E. Focardi ${ }^{a, b}$, S. Frosali ${ }^{a}$ b, E. Gallo ${ }^{a}$, C. Genta $^{a}$, P. Lenzi ${ }^{\text {a,b }}$, M. Meschini ${ }^{\text {a }}$, S. Paoletti ${ }^{\text {a }}$, G. Sguazzoni ${ }^{\text {a }}$, A. Tropiano ${ }^{\mathrm{a}, 1}$

a INFN Sezione di Firenze, Firenze, Italy

${ }^{\mathrm{b}}$ Università di Firenze, Firenze, Italy

L. Benussi, S. Bianco, S. Colafranceschi ${ }^{14}$, F. Fabbri, D. Piccolo

INFN Laboratori Nazionali di Frascati, Frascati, Italy

P. Fabbricatore, R. Musenich

INFN Sezione di Genova, Genova, Italy

A. Benaglia ${ }^{a, b}$, F. De Guio a,b,1, L. Di Matteo ${ }^{a, b}$, A. Ghezzi ${ }^{a, b, 1}$, M. Malberti $^{a, b}$, S. Malvezzi ${ }^{a}$, A. Martelli ${ }^{a, b}$, A. Massironi ${ }^{a, b}$, D. Menasce ${ }^{a}$, L. Moroni $^{a}$, M. Paganoni ${ }^{a, b}$, D. Pedrini ${ }^{\text {a }}$, S. Ragazzi $^{\text {a,b }}{ }^{\text {, }}$ N. Redaelli ${ }^{\text {a }, ~ S . ~ S a l a ~}{ }^{\text {a }}$ T. Tabarelli de Fatis ${ }^{a, b}$, V. Tancini ${ }^{a, b}$

a INFN Sezione di Milano-Biccoca, Milano, Italy

${ }^{\mathrm{b}}$ Università di Milano-Bicocca, Milano, Italy

S. Buontempo ${ }^{a}$, C.A. Carrillo Montoya ${ }^{a}$, A. Cimmino ${ }^{a, b}$, A. De Cosa ${ }^{a, b}$, M. De Gruttola ${ }^{a, b}$, F. Fabozzi ${ }^{a, 15}$,

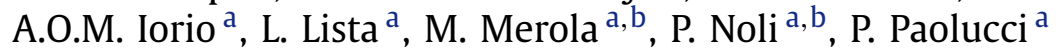

a INFN Sezione di Napoli, Napoli, Italy

b Università di Napoli “Federico II”, Napoli, Italy

P. Azzi ${ }^{\text {a }}$, N. Bacchetta ${ }^{\text {a }}$, P. Bellan ${ }^{a, b}$, D. Bisello ${ }^{a, b}$, A. Branca ${ }^{a}$, R. Carlin ${ }^{a, b}$, P. Checchia ${ }^{a}$, E. Conti $^{a}$,

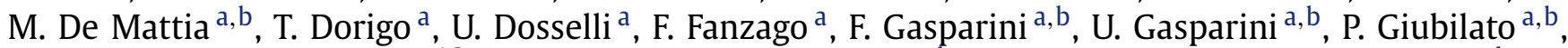
A. Gresele ${ }^{a, c}$, S. Lacaprara ${ }^{a, 16}$, I. Lazzizzera ${ }^{a, c}$, M. Margoni ${ }^{a, b}$, M. Mazzucato ${ }^{a}$, A.T. Meneguzzo ${ }^{a, b}$, L. Perrozzi $^{a, 1}$, N. Pozzobon ${ }^{a, b}$, P. Ronchese ${ }^{a, b}$, F. Simonetto ${ }^{a, b}$, E. Torassa ${ }^{a}$, M. Tosi ${ }^{a, b}$, S. Vanini ${ }^{a, b}$, P. Zotto ${ }^{\mathrm{a}, \mathrm{b}}, \mathrm{G}$. Zumerle $\mathrm{a}^{\mathrm{a}, \mathrm{b}}$

a INFN Sezione di Padova, Padova, Italy

b Università di Padova, Padova, Italy

c Università di Trento (Trento), Padova, Italy

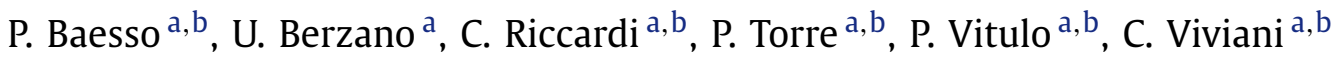

a INFN Sezione di Pavia, Pavia, Italy

b Università di Pavia, Pavia, Italy 
M. Biasini ${ }^{\mathrm{a}, \mathrm{b}}$, G.M. Bilei ${ }^{\mathrm{a}}$, B. Caponeri ${ }^{\mathrm{a}, \mathrm{b}}$, L. Fanò ${ }^{\mathrm{a}, \mathrm{b}}$, P. Lariccia ${ }^{\mathrm{a}, \mathrm{b}}$, A. Lucaroni $^{\mathrm{a}, \mathrm{b}, 1}$, G. Mantovani $^{\mathrm{a}, \mathrm{b}}$,

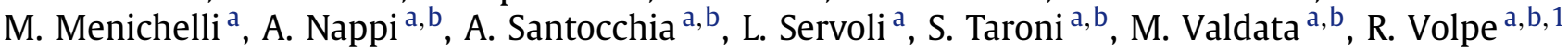

a INFN Sezione di Perugia, Perugia, Italy

${ }^{\mathrm{b}}$ Università di Perugia, Perugia, Italy

P. Azzurri ${ }^{a, c}$, G. Bagliesi ${ }^{a}$, J. Bernardini ${ }^{a, b}$, T. Boccali ${ }^{a, 1}$, G. Broccolo ${ }^{a, c}$, R. Castaldi $^{\text {a }}$, R.T. D'Agnolo ${ }^{a, c}$,

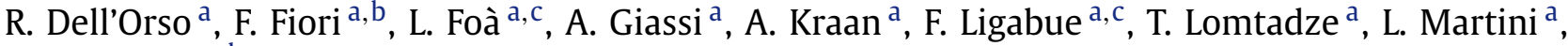
A. Messineo ${ }^{\text {a,b }}$, F. Palla ${ }^{\text {a }}$, F. Palmonari ${ }^{a}$, S. Sarkar ${ }^{\text {a,c }}$, G. Segneri ${ }^{a}$, A.T. Serban ${ }^{\text {a }}$, P. Spagnolo ${ }^{\text {a }}$, R. Tenchini ${ }^{\text {a,* }}$, G. Tonelli ${ }^{\text {a,b, }}{ }^{\text {, A. Venturi }}{ }^{\text {a, }}$, P.G. Verdini ${ }^{\text {a }}$

a INFN Sezione di Pisa, Pisa, Italy

b Università di Pisa, Pisa, Italy

c Scuola Normale Superiore di Pisa, Pisa, Italy

L. Barone ${ }^{a, b}$, F. Cavallari a , D. Del Re ${ }^{a, b}$, E. Di Marco ${ }^{a, b}$, M. Diemoz ${ }^{a}$, D. Franci ${ }^{a, b}$, M. Grassi ${ }^{\text {a }}$, E. Longo ${ }^{a, b}$, G. Organtini ${ }^{a, b}$, A. Palma ${ }^{a, b}$, F. Pandolfi ${ }^{a, b, 1}$, R. Paramatti ${ }^{a}$, S. Rahatlou ${ }^{a, b}$

a INFN Sezione di Roma, Roma, Italy

b Università di Roma “La Sapienza”, Roma, Italy

N. Amapane ${ }^{\mathrm{a}, \mathrm{b}}$, R. Arcidiacono ${ }^{\mathrm{a}, \mathrm{c}}, \mathrm{S}$. Argiro ${ }^{\mathrm{a}, \mathrm{b}}$, M. Arneodo ${ }^{\mathrm{a}, \mathrm{c}}$, C. Biino ${ }^{\mathrm{a}}$, C. Botta $^{\mathrm{a}, \mathrm{b}, 1}$, N. Cartiglia ${ }^{\mathrm{a}}$, R. Castello ${ }^{a, b}$, M. Costa $^{a, b}$, N. Demaria ${ }^{a}$, A. Graziano ${ }^{a, b}, 1$, C. Mariotti ${ }^{a}$, M. Marone ${ }^{a, b}$, S. Maselli ${ }^{a}$,

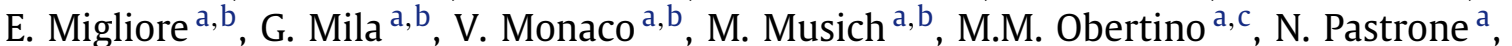

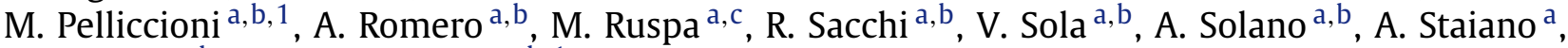
D. Trocino ${ }^{a, b}, A$. Vilela Pereira ${ }^{a, b, 1}$

a INFN Sezione di Torino, Torino, Italy

b Università di Torino, Torino, Italy

c Università del Piemonte Orientale (Novara), Torino, Italy

F. Ambroglini ${ }^{a, b}$, S. Belforte ${ }^{a}$, F. Cossutti ${ }^{a}$, G. Della Ricca ${ }^{a, b}$, B. Gobbo $^{a}$, D. Montanino $^{\mathrm{a}, \mathrm{b}}$, A. Penzo a

a INFN Sezione di Trieste, Trieste, Italy

b Università di Trieste, Trieste, Italy

S.G. Heo

Kangwon National University, Chunchon, Republic of Korea

S. Chang, J. Chung, D.H. Kim, G.N. Kim, J.E. Kim, D.J. Kong, H. Park, D. Son, D.C. Son

Kyungpook National University, Daegu, Republic of Korea

Zero Kim, J.Y. Kim, S. Song

Chonnam National University, Institute for Universe and Elementary Particles, Kwangju, Republic of Korea

S. Choi, B. Hong, M. Jo, H. Kim, J.H. Kim, T.J. Kim, K.S. Lee, D.H. Moon, S.K. Park, H.B. Rhee, E. Seo, S. Shin, K.S. Sim

Korea University, Seoul, Republic of Korea

M. Choi, S. Kang, H. Kim, C. Park, I.C. Park, S. Park, G. Ryu

University of Seoul, Seoul, Republic of Korea

Y. Choi, Y.K. Choi, J. Goh, J. Lee, S. Lee, H. Seo, I. Yu

Sungkyunkwan University, Suwon, Republic of Korea

M.J. Bilinskas, I. Grigelionis, M. Janulis, D. Martisiute, P. Petrov, T. Sabonis 
H. Castilla Valdez, E. De La Cruz Burelo, R. Lopez-Fernandez, A. Sánchez Hernández, L.M. Villasenor-Cendejas

Centro de Investigacion y de Estudios Avanzados del IPN, Mexico City, Mexico

S. Carrillo Moreno, F. Vazquez Valencia

Universidad Iberoamericana, Mexico City, Mexico

\section{H.A. Salazar Ibarguen}

Benemerita Universidad Autonoma de Puebla, Puebla, Mexico

E. Casimiro Linares, A. Morelos Pineda, M.A. Reyes-Santos

Universidad Autónoma de San Luis Potosí, San Luis Potosí, Mexico

P. Allfrey, D. Krofcheck

University of Auckland, Auckland, New Zealand

P.H. Butler, R. Doesburg, H. Silverwood

University of Canterbury, Christchurch, New Zealand

M. Ahmad, I. Ahmed, M.I. Asghar, H.R. Hoorani, W.A. Khan, T. Khurshid, S. Qazi

National Centre for Physics, Quaid-I-Azam University, Islamabad, Pakistan

M. Cwiok, W. Dominik, K. Doroba, A. Kalinowski, M. Konecki, J. Krolikowski

Institute of Experimental Physics, Faculty of Physics, University of Warsaw, Warsaw, Poland

T. Frueboes, R. Gokieli, M. Górski, M. Kazana, K. Nawrocki, K. Romanowska-Rybinska, M. Szleper,

G. Wrochna, P. Zalewski

Soltan Institute for Nuclear Studies, Warsaw, Poland

N. Almeida, A. David, P. Faccioli, P.G. Ferreira Parracho, M. Gallinaro, P. Martins, P. Musella, A. Nayak, P.Q. Ribeiro, J. Seixas, P. Silva, J. Varela ${ }^{1}$, H.K. Wöhri

Laboratório de Instrumentação e Física Experimental de Partículas, Lisboa, Portugal

I. Belotelov, P. Bunin, M. Finger, M. Finger Jr., I. Golutvin, A. Kamenev, V. Karjavin, G. Kozlov,

A. Lanev, P. Moisenz, V. Palichik, V. Perelygin, S. Shmatov, V. Smirnov, A. Volodko,

A. Zarubin

Joint Institute for Nuclear Research, Dubna, Russia

N. Bondar, V. Golovtsov, Y. Ivanov, V. Kim, P. Levchenko, V. Murzin, V. Oreshkin, I. Smirnov, V. Sulimov, L. Uvarov, S. Vavilov, A. Vorobyev

Petersburg Nuclear Physics Institute, Gatchina (St. Petersburg), Russia

Yu. Andreev, S. Gninenko, N. Golubev, M. Kirsanov, N. Krasnikov, V. Matveev, A. Pashenkov, A. Toropin, S. Troitsky

Institute for Nuclear Research, Moscow, Russia

V. Epshteyn, V. Gavrilov, V. Kaftanov ${ }^{\dagger}$, M. Kossov ${ }^{1}$, A. Krokhotin, N. Lychkovskaya, G. Safronov, S. Semenov, V. Stolin, E. Vlasov, A. Zhokin 
E. Boos, M. Dubinin ${ }^{17}$, L. Dudko, A. Ershov, A. Gribushin, O. Kodolova, I. Lokhtin, S. Obraztsov, S. Petrushanko, L. Sarycheva, V. Savrin, A. Snigirev

Moscow State University, Moscow, Russia

V. Andreev, M. Azarkin, I. Dremin, M. Kirakosyan, S.V. Rusakov, A. Vinogradov

P.N. Lebedev Physical Institute, Moscow, Russia

I. Azhgirey, S. Bitioukov, V. Grishin ${ }^{1}$, V. Kachanov, D. Konstantinov, A. Korablev, V. Krychkine, V. Petrov, R. Ryutin, S. Slabospitsky, A. Sobol, L. Tourtchanovitch, S. Troshin, N. Tyurin, A. Uzunian, A. Volkov

State Research Center of Russian Federation, Institute for High Energy Physics, Protvino, Russia

P. Adzic ${ }^{18}$, M. Djordjevic, D. Krpic ${ }^{18}$, J. Milosevic

University of Belgrade, Faculty of Physics and Vinca Institute of Nuclear Sciences, Belgrade, Serbia

M. Aguilar-Benitez, J. Alcaraz Maestre, P. Arce, C. Battilana, E. Calvo, M. Cepeda, M. Cerrada, N. Colino, B. De La Cruz, C. Diez Pardos, D. Domínguez Vázquez, C. Fernandez Bedoya, J.P. Fernández Ramos,

A. Ferrando, J. Flix, M.C. Fouz, P. Garcia-Abia, O. Gonzalez Lopez, S. Goy Lopez, J.M. Hernandez, M.I. Josa, G. Merino, J. Puerta Pelayo, I. Redondo, L. Romero, J. Santaolalla, C. Willmott

Centro de Investigaciones Energéticas Medioambientales y Tecnológicas (CIEMAT), Madrid, Spain

C. Albajar, G. Codispoti, J.F. de Trocóniz

Universidad Autónoma de Madrid, Madrid, Spain

J. Cuevas, J. Fernandez Menendez, S. Folgueras, I. Gonzalez Caballero, L. Lloret Iglesias, J.M. Vizan Garcia Universidad de Oviedo, Oviedo, Spain

J.A. Brochero Cifuentes, I.J. Cabrillo, A. Calderon, M. Chamizo Llatas, S.H. Chuang, J. Duarte Campderros, M. Felcini ${ }^{19}$, M. Fernandez, G. Gomez, J. Gonzalez Sanchez, C. Jorda, P. Lobelle Pardo, A. Lopez Virto, J. Marco, R. Marco, C. Martinez Rivero, F. Matorras, F.J. Munoz Sanchez, J. Piedra Gomez ${ }^{20}$, T. Rodrigo, A. Ruiz Jimeno, L. Scodellaro, M. Sobron Sanudo, I. Vila, R. Vilar Cortabitarte

Instituto de Física de Cantabria (IFCA), CSIC - Universidad de Cantabria, Santander, Spain

D. Abbaneo, E. Auffray, G. Auzinger, P. Baillon, A.H. Ball, D. Barney, A.J. Bell ${ }^{21}$, D. Benedetti, C. Bernet ${ }^{3}$, W. Bialas, P. Bloch, A. Bocci, S. Bolognesi, H. Breuker, G. Brona, K. Bunkowski, T. Camporesi, E. Cano, G. Cerminara, T. Christiansen, J.A. Coarasa Perez, B. Curé, D. D’Enterria, A. De Roeck, F. Duarte Ramos, A. Elliott-Peisert, B. Frisch, W. Funk, A. Gaddi, S. Gennai, G. Georgiou, H. Gerwig, D. Gigi, K. Gill, D. Giordano, F. Glege, R. Gomez-Reino Garrido, M. Gouzevitch, P. Govoni, S. Gowdy, L. Guiducci, M. Hansen, J. Harvey, J. Hegeman, B. Hegner, C. Henderson, G. Hesketh, H.F. Hoffmann, A. Honma, V. Innocente, P. Janot, E. Karavakis, P. Lecoq, C. Leonidopoulos, C. Lourenço, A. Macpherson, T. Mäki, L. Malgeri, M. Mannelli, L. Masetti, F. Meijers, S. Mersi, E. Meschi, R. Moser, M.U. Mozer, M. Mulders, E. Nesvold ${ }^{1}$, M. Nguyen, T. Orimoto, L. Orsini, E. Perez, A. Petrilli, A. Pfeiffer, M. Pierini, M. Pimiä, G. Polese, A. Racz, G. Rolandi ${ }^{22}$, T. Rommerskirchen, C. Rovelli ${ }^{23}$, M. Rovere, H. Sakulin, C. Schäfer, C. Schwick, I. Segoni, A. Sharma, P. Siegrist, M. Simon, P. Sphicas ${ }^{24}$, D. Spiga, M. Spiropulu ${ }^{17}$, F. Stöckli, M. Stoye, P. Tropea, A. Tsirou, A. Tsyganov, G.I. Veres ${ }^{11}$, P. Vichoudis, M. Voutilainen, W.D. Zeuner

CERN, European Organization for Nuclear Research, Geneva, Switzerland

W. Bertl, K. Deiters, W. Erdmann, K. Gabathuler, R. Horisberger, Q. Ingram, H.C. Kaestli, S. König, D. Kotlinski, U. Langenegger, F. Meier, D. Renker, T. Rohe, J. Sibille ${ }^{25}$, A. Starodumov 26 
P. Bortignon, L. Caminada ${ }^{27}$, Z. Chen, S. Cittolin, G. Dissertori, M. Dittmar, J. Eugster, K. Freudenreich, C. Grab, A. Hervé, W. Hintz, P. Lecomte, W. Lustermann, C. Marchica 27, P. Martinez Ruiz del Arbol, P. Meridiani, P. Milenovic ${ }^{28}$, F. Moortgat, P. Nef, F. Nessi-Tedaldi, L. Pape, F. Pauss, T. Punz, A. Rizzi, F.J. Ronga, M. Rossini, L. Sala, A.K. Sanchez, M.-C. Sawley, B. Stieger, L. Tauscher ${ }^{\dagger}$, A. Thea, K. Theofilatos, D. Treille, C. Urscheler, R. Wallny ${ }^{19}$, M. Weber, L. Wehrli, J. Weng

Institute for Particle Physics, ETH Zurich, Zurich, Switzerland

E. Aguiló, C. Amsler, V. Chiochia, S. De Visscher, C. Favaro, M. Ivova Rikova, B. Millan Mejias, C. Regenfus, P. Robmann, A. Schmidt, H. Snoek, L. Wilke

Universität Zürich, Zurich, Switzerland

Y.H. Chang, K.H. Chen, W.T. Chen, S. Dutta, A. Go, C.M. Kuo, S.W. Li, W. Lin, M.H. Liu, Z.K. Liu, Y.J. Lu, J.H. Wu, S.S. Yu

National Central University, Chung-Li, Taiwan

P. Bartalini, P. Chang, Y.H. Chang, Y.W. Chang, Y. Chao, K.F. Chen, W.-S. Hou, Y. Hsiung, K.Y. Kao, Y.J. Lei, R.-S. Lu, J.G. Shiu, Y.M. Tzeng, M. Wang

National Taiwan University (NTU), Taipei, Taiwan

A. Adiguzel, M.N. Bakirci, S. Cerci ${ }^{29}$, Z. Demir, C. Dozen, I. Dumanoglu, E. Eskut, S. Girgis, G. Gokbulut, Y. Guler, E. Gurpinar, I. Hos, E.E. Kangal, T. Karaman, A. Kayis Topaksu, A. Nart, G. Onengut, K. Ozdemir, S. Ozturk, A. Polatoz, K. Sogut ${ }^{30}$, B. Tali, H. Topakli, D. Uzun, L.N. Vergili, M. Vergili, C. Zorbilmez

Cukurova University, Adana, Turkey

I.V. Akin, T. Aliev, S. Bilmis, M. Deniz, H. Gamsizkan, A.M. Guler, K. Ocalan, A. Ozpineci, M. Serin, R. Sever, U.E. Surat, E. Yildirim, M. Zeyrek

Middle East Technical University, Physics Department, Ankara, Turkey

M. Deliomeroglu, D. Demir ${ }^{31}$, E. Gülmez, A. Halu, B. Isildak, M. Kaya ${ }^{32}$, O. Kaya ${ }^{32}$, S. Ozkorucuklu ${ }^{33}$, N. Sonmez ${ }^{34}$

Bogazici University, Istanbul, Turkey

L. Levchuk

National Scientific Center, Kharkov Institute of Physics and Technology, Kharkov, Ukraine

P. Bell, F. Bostock, J.J. Brooke, T.L. Cheng, E. Clement, D. Cussans, R. Frazier, J. Goldstein, M. Grimes, M. Hansen, D. Hartley, G.P. Heath, H.F. Heath, B. Huckvale, J. Jackson, L. Kreczko, S. Metson, D.M. Newbold ${ }^{35}$, K. Nirunpong, A. Poll, S. Senkin, V.J. Smith, S. Ward

University of Bristol, Bristol, United Kingdom

L. Basso, K.W. Bell, A. Belyaev, C. Brew, R.M. Brown, B. Camanzi, D.J.A. Cockerill, J.A. Coughlan, K. Harder, S. Harper, B.W. Kennedy, E. Olaiya, D. Petyt, B.C. Radburn-Smith, C.H. Shepherd-Themistocleous, I.R. Tomalin, W.J. Womersley, S.D. Worm

Rutherford Appleton Laboratory, Didcot, United Kingdom

R. Bainbridge, G. Ball, J. Ballin, R. Beuselinck, O. Buchmuller, D. Colling, N. Cripps, M. Cutajar, G. Davies, M. Della Negra, J. Fulcher, D. Futyan, A. Guneratne Bryer, G. Hall, Z. Hatherell, J. Hays, G. Iles, G. Karapostoli, L. Lyons, A.-M. Magnan, J. Marrouche, R. Nandi, J. Nash, A. Nikitenko ${ }^{26}$, A. Papageorgiou, M. Pesaresi, K. Petridis, M. Pioppi ${ }^{36}$, D.M. Raymond, N. Rompotis, A. Rose, M.J. Ryan, C. Seez, P. Sharp, A. Sparrow, A. Tapper, S. Tourneur, M. Vazquez Acosta, T. Virdee, S. Wakefield, D. Wardrope, T. Whyntie 
M. Barrett, M. Chadwick, J.E. Cole, P.R. Hobson, A. Khan, P. Kyberd, D. Leslie, W. Martin, I.D. Reid, L. Teodorescu

Brunel University, Uxbridge, United Kingdom

\section{K. Hatakeyama}

Baylor University, Waco, USA

T. Bose, E. Carrera Jarrin, A. Clough, C. Fantasia, A. Heister, J.St. John, P. Lawson, D. Lazic, J. Rohlf, D. Sperka, L. Sulak

Boston University, Boston, USA

A. Avetisyan, S. Bhattacharya, J.P. Chou, D. Cutts, A. Ferapontov, U. Heintz, S. Jabeen, G. Kukartsev, G. Landsberg, M. Narain, D. Nguyen, M. Segala, T. Speer, K.V. Tsang

Brown University, Providence, USA

M.A. Borgia, R. Breedon, M. Calderon De La Barca Sanchez, D. Cebra, S. Chauhan, M. Chertok, J. Conway, P.T. Cox, J. Dolen, R. Erbacher, E. Friis, W. Ko, A. Kopecky, R. Lander, H. Liu, S. Maruyama, T. Miceli, M. Nikolic, D. Pellett, J. Robles, S. Salur, T. Schwarz, M. Searle, J. Smith, M. Squires, M. Tripathi, R. Vasquez Sierra, C. Veelken

University of California, Davis, Davis, USA

V. Andreev, K. Arisaka, D. Cline, R. Cousins, A. Deisher, J. Duris, S. Erhan, C. Farrell, J. Hauser, M. Ignatenko, C. Jarvis, C. Plager, G. Rakness, P. Schlein ${ }^{\dagger}$, J. Tucker, V. Valuev

University of California, Los Angeles, Los Angeles, USA

J. Babb, R. Clare, J. Ellison, J.W. Gary, F. Giordano, G. Hanson, G.Y. Jeng, S.C. Kao, F. Liu, H. Liu, A. Luthra, H. Nguyen, G. Pasztor ${ }^{37}$, A. Satpathy, B.C. Shen ${ }^{\dagger}$, R. Stringer, J. Sturdy, S. Sumowidagdo, R. Wilken, S. Wimpenny

University of California, Riverside, Riverside, USA

W. Andrews, J.G. Branson, G.B. Cerati, E. Dusinberre, D. Evans, F. Golf, A. Holzner, R. Kelley, M. Lebourgeois, J. Letts, B. Mangano, J. Muelmenstaedt, S. Padhi, C. Palmer, G. Petrucciani, H. Pi, M. Pieri, R. Ranieri, M. Sani, V. Sharma ${ }^{1}$, S. Simon, Y. Tu, A. Vartak, F. Würthwein, A. Yagil

University of California, San Diego, La Jolla, USA

D. Barge, R. Bellan, C. Campagnari, M. D’Alfonso, T. Danielson, K. Flowers, P. Geffert, J. Incandela, C. Justus, P. Kalavase, S.A. Koay, D. Kovalskyi, V. Krutelyov, S. Lowette, N. Mccoll, V. Pavlunin, F. Rebassoo, J. Ribnik, J. Richman, R. Rossin, D. Stuart, W. To, J.R. Vlimant

University of California, Santa Barbara, Santa Barbara, USA

A. Bornheim, J. Bunn, Y. Chen, M. Gataullin, D. Kcira, V. Litvine, Y. Ma, A. Mott, H.B. Newman, C. Rogan, V. Timciuc, P. Traczyk, J. Veverka, R. Wilkinson, Y. Yang, R.Y. Zhu

California Institute of Technology, Pasadena, USA

B. Akgun, R. Carroll, T. Ferguson, Y. Iiyama, D.W. Jang, S.Y. Jun, Y.F. Liu, M. Paulini, J. Russ, N. Terentyev, H. Vogel, I. Vorobiev

Carnegie Mellon University, Pittsburgh, USA

J.P. Cumalat, M.E. Dinardo, B.R. Drell, C.J. Edelmaier, W.T. Ford, B. Heyburn, E. Luiggi Lopez, U. Nauenberg, J.G. Smith, K. Stenson, K.A. Ulmer, S.R. Wagner, S.L. Zang 
L. Agostino, J. Alexander, A. Chatterjee, S. Das, N. Eggert, L.J. Fields, L.K. Gibbons, B. Heltsley, W. Hopkins, A. Khukhunaishvili, B. Kreis, V. Kuznetsov, G. Nicolas Kaufman, J.R. Patterson, D. Puigh, D. Riley, A. Ryd, X. Shi, W. Sun, W.D. Teo, J. Thom, J. Thompson, J. Vaughan, Y. Weng, L. Winstrom, P. Wittich

Cornell University, Ithaca, USA

A. Biselli, G. Cirino, D. Winn

Fairfield University, Fairfield, USA

S. Abdullin, M. Albrow, J. Anderson, G. Apollinari, M. Atac, J.A. Bakken, S. Banerjee, L.A.T. Bauerdick, A. Beretvas, J. Berryhill, P.C. Bhat, I. Bloch, F. Borcherding, K. Burkett, J.N. Butler, V. Chetluru, H.W.K. Cheung, F. Chlebana, S. Cihangir, M. Demarteau, D.P. Eartly, V.D. Elvira, S. Esen, I. Fisk, J. Freeman, Y. Gao, E. Gottschalk, D. Green, K. Gunthoti, O. Gutsche, A. Hahn, J. Hanlon, R.M. Harris, J. Hirschauer, B. Hooberman, E. James, H. Jensen, M. Johnson, U. Joshi, R. Khatiwada, B. Kilminster, B. Klima, K. Kousouris, S. Kunori, S. Kwan, P. Limon, R. Lipton, J. Lykken, K. Maeshima, J.M. Marraffino, D. Mason, P. McBride, T. McCauley, T. Miao, K. Mishra, S. Mrenna, Y. Musienko ${ }^{38}$, C. Newman-Holmes, V. O’Dell, S. Popescu ${ }^{39}$, R. Pordes, O. Prokofyev, N. Saoulidou, E. Sexton-Kennedy, S. Sharma, A. Soha, W.J. Spalding, L. Spiegel, P. Tan, L. Taylor, S. Tkaczyk, L. Uplegger, E.W. Vaandering, R. Vidal, J. Whitmore, W. Wu, F. Yang, F. Yumiceva, J.C. Yun

Fermi National Accelerator Laboratory, Batavia, USA

D. Acosta, P. Avery, D. Bourilkov, M. Chen, G.P. Di Giovanni, D. Dobur, A. Drozdetskiy, R.D. Field, M. Fisher, Y. Fu, I.K. Furic, J. Gartner, S. Goldberg, B. Kim, S. Klimenko, J. Konigsberg, A. Korytov, A. Kropivnitskaya, T. Kypreos, K. Matchev, G. Mitselmakher, L. Muniz, Y. Pakhotin, C. Prescott, R. Remington, M. Schmitt, B. Scurlock, P. Sellers, N. Skhirtladze, D. Wang, J. Yelton, M. Zakaria University of Florida, Gainesville, USA

C. Ceron, V. Gaultney, L. Kramer, L.M. Lebolo, S. Linn, P. Markowitz, G. Martinez, J.L. Rodriguez Florida International University, Miami, USA

T. Adams, A. Askew, D. Bandurin, J. Bochenek, J. Chen, B. Diamond, S.V. Gleyzer, J. Haas, S. Hagopian, V. Hagopian, M. Jenkins, K.F. Johnson, H. Prosper, S. Sekmen, V. Veeraraghavan

Florida State University, Tallahassee, USA

M.M. Baarmand, B. Dorney, S. Guragain, M. Hohlmann, H. Kalakhety, R. Ralich, I. Vodopiyanov Florida Institute of Technology, Melbourne, USA

M.R. Adams, I.M. Anghel, L. Apanasevich, Y. Bai, V.E. Bazterra, R.R. Betts, J. Callner, R. Cavanaugh, C. Dragoiu, E.J. Garcia-Solis, C.E. Gerber, D.J. Hofman, S. Khalatyan, F. Lacroix, C. O’Brien, C. Silvestre, A. Smoron, D. Strom, N. Varelas

University of Illinois at Chicago (UIC), Chicago, USA

U. Akgun, E.A. Albayrak, B. Bilki, K. Cankocak ${ }^{40}$, W. Clarida, F. Duru, C.K. Lae, E. McCliment, J.-P. Merlo, H. Mermerkaya, A. Mestvirishvili, A. Moeller, J. Nachtman, C.R. Newsom, E. Norbeck, J. Olson, Y. Onel, F. Ozok, S. Sen, J. Wetzel, T. Yetkin, K. Yi

The University of Iowa, Iowa City, USA

B.A. Barnett, B. Blumenfeld, A. Bonato, C. Eskew, D. Fehling, G. Giurgiu, A.V. Gritsan, Z.J. Guo, G. Hu, P. Maksimovic, S. Rappoccio, M. Swartz, N.V. Tran, A. Whitbeck 
P. Baringer, A. Bean, G. Benelli, O. Grachov, M. Murray, D. Noonan, V. Radicci, S. Sanders, J.S. Wood, V. Zhukova

The University of Kansas, Lawrence, USA

T. Bolton, I. Chakaberia, A. Ivanov, M. Makouski, Y. Maravin, S. Shrestha, I. Svintradze, Z. Wan

Kansas State University, Manhattan, USA

J. Gronberg, D. Lange, D. Wright

Lawrence Livermore National Laboratory, Livermore, USA

A. Baden, M. Boutemeur, S.C. Eno, D. Ferencek, J.A. Gomez, N.J. Hadley, R.G. Kellogg, M. Kirn, Y. Lu, A.C. Mignerey, K. Rossato, P. Rumerio, F. Santanastasio, A. Skuja, J. Temple, M.B. Tonjes, S.C. Tonwar, E. Twedt

University of Maryland, College Park, USA

B. Alver, G. Bauer, J. Bendavid, W. Busza, E. Butz, I.A. Cali, M. Chan, V. Dutta, P. Everaerts,

G. Gomez Ceballos, M. Goncharov, K.A. Hahn, P. Harris, Y. Kim, M. Klute, Y.-J. Lee, W. Li, C. Loizides, P.D. Luckey, T. Ma, S. Nahn, C. Paus, D. Ralph, C. Roland, G. Roland, M. Rudolph, G.S.F. Stephans, K. Sumorok, K. Sung, E.A. Wenger, S. Xie, M. Yang, Y. Yilmaz, A.S. Yoon, M. Zanetti

Massachusetts Institute of Technology, Cambridge, USA

P. Cole, S.I. Cooper, P. Cushman, B. Dahmes, A. De Benedetti, P.R. Dudero, G. Franzoni, J. Haupt, K. Klapoetke, Y. Kubota, J. Mans, V. Rekovic, R. Rusack, M. Sasseville, A. Singovsky

University of Minnesota, Minneapolis, USA

L.M. Cremaldi, R. Godang, R. Kroeger, L. Perera, R. Rahmat, D.A. Sanders, D. Summers

University of Mississippi, University, USA

K. Bloom, S. Bose, J. Butt, D.R. Claes, A. Dominguez, M. Eads, J. Keller, T. Kelly, I. Kravchenko, J. Lazo-Flores, C. Lundstedt, H. Malbouisson, S. Malik, G.R. Snow

University of Nebraska-Lincoln, Lincoln, USA

U. Baur, A. Godshalk, I. Iashvili, S. Jain, A. Kharchilava, A. Kumar, S.P. Shipkowski, K. Smith

State University of New York at Buffalo, Buffalo, USA

G. Alverson, E. Barberis, D. Baumgartel, O. Boeriu, M. Chasco, K. Kaadze, S. Reucroft, J. Swain, D. Wood, J. Zhang

Northeastern University, Boston, USA

A. Anastassov, A. Kubik, N. Odell, R.A. Ofierzynski, B. Pollack, A. Pozdnyakov, M. Schmitt, S. Stoynev, M. Velasco, S. Won

Northwestern University, Evanston, USA

L. Antonelli, D. Berry, M. Hildreth, C. Jessop, D.J. Karmgard, J. Kolb, T. Kolberg, K. Lannon, W. Luo, S. Lynch, N. Marinelli, D.M. Morse, T. Pearson, R. Ruchti, J. Slaunwhite, N. Valls, J. Warchol, M. Wayne, J. Ziegler

University of Notre Dame, Notre Dame, USA

B. Bylsma, L.S. Durkin, J. Gu, C. Hill, P. Killewald, K. Kotov, T.Y. Ling, M. Rodenburg, G. Williams 
N. Adam, E. Berry, P. Elmer, D. Gerbaudo, V. Halyo, P. Hebda, A. Hunt, J. Jones, E. Laird, D. Lopes Pegna, D. Marlow, T. Medvedeva, M. Mooney, J. Olsen, P. Piroué, X. Quan, H. Saka, D. Stickland, C. Tully, J.S. Werner, A. Zuranski

Princeton University, Princeton, USA

J.G. Acosta, X.T. Huang, A. Lopez, H. Mendez, S. Oliveros, J.E. Ramirez Vargas, A. Zatserklyaniy University of Puerto Rico, Mayaguez, USA

E. Alagoz, V.E. Barnes, G. Bolla, L. Borrello, D. Bortoletto, A. Everett, A.F. Garfinkel, Z. Gecse, L. Gutay, Z. Hu, M. Jones, O. Koybasi, A.T. Laasanen, N. Leonardo, C. Liu, V. Maroussov, P. Merkel, D.H. Miller, N. Neumeister, I. Shipsey, D. Silvers, A. Svyatkovskiy, H.D. Yoo, J. Zablocki, Y. Zheng

Purdue University, West Lafayette, USA

P. Jindal, N. Parashar

Purdue University Calumet, Hammond, USA

C. Boulahouache, V. Cuplov, K.M. Ecklund, F.J.M. Geurts, J.H. Liu, B.P. Padley, R. Redjimi, J. Roberts, J. Zabel

Rice University, Houston, USA

B. Betchart, A. Bodek, Y.S. Chung, R. Covarelli, P. de Barbaro, R. Demina, Y. Eshaq, H. Flacher, A. Garcia-Bellido, P. Goldenzweig, Y. Gotra, J. Han, A. Harel, D.C. Miner, D. Orbaker, G. Petrillo, D. Vishnevskiy, M. Zielinski

University of Rochester, Rochester, USA

A. Bhatti, L. Demortier, K. Goulianos, G. Lungu, C. Mesropian, M. Yan

The Rockefeller University, New York, USA

O. Atramentov, A. Barker, D. Duggan, Y. Gershtein, R. Gray, E. Halkiadakis, D. Hidas, D. Hits, A. Lath, S. Panwalkar, R. Patel, A. Richards, K. Rose, S. Schnetzer, S. Somalwar, R. Stone, S. Thomas

Rutgers, the State University of New Jersey, Piscataway, USA

G. Cerizza, M. Hollingsworth, S. Spanier, Z.C. Yang, A. York

University of Tennessee, Knoxville, USA

J. Asaadi, R. Eusebi, J. Gilmore, A. Gurrola, T. Kamon, V. Khotilovich, R. Montalvo, C.N. Nguyen,

I. Osipenkov, J. Pivarski, A. Safonov, S. Sengupta, A. Tatarinov, D. Toback, M. Weinberger

Texas A\&'M University, College Station, USA

N. Akchurin, C. Bardak, J. Damgov, C. Jeong, K. Kovitanggoon, S.W. Lee, P. Mane, Y. Roh, A. Sill, I. Volobouev, R. Wigmans, E. Yazgan

Texas Tech University, Lubbock, USA

E. Appelt, E. Brownson, D. Engh, C. Florez, W. Gabella, W. Johns, P. Kurt, C. Maguire, A. Melo, P. Sheldon, J. Velkovska

Vanderbilt University, Nashville, USA

M.W. Arenton, M. Balazs, S. Boutle, M. Buehler, S. Conetti, B. Cox, B. Francis, R. Hirosky, A. Ledovskoy, C. Lin, C. Neu, R. Yohay 


\section{S. Gollapinni, R. Harr, P.E. Karchin, P. Lamichhane, M. Mattson, C. Milstène, A. Sakharov}

Wayne State University, Detroit, USA

\section{Anderson, M. Bachtis, J.N. Bellinger, D. Carlsmith, S. Dasu, J. Efron, L. Gray, K.S. Grogg, M. Grothe, R. Hall-Wilton ${ }^{1}$, M. Herndon, P. Klabbers, J. Klukas, A. Lanaro, C. Lazaridis, J. Leonard, D. Lomidze, R. Loveless, A. Mohapatra, D. Reeder, I. Ross, A. Savin, W.H. Smith, J. Swanson, M. Weinberg}

University of Wisconsin, Madison, USA

* Corresponding author.

E-mail address: Roberto.Tenchini@cern.ch (R. Tenchini).

$\dagger$ Deceased.

1 Also at CERN, European Organization for Nuclear Research, Geneva, Switzerland.

2 Also at Universidade Federal do ABC, Santo Andre, Brazil.

3 Also at Laboratoire Leprince-Ringuet, Ecole Polytechnique, IN2P3-CNRS, Palaiseau, France.

4 Also at Suez Canal University, Suez, Egypt.

5 Also at Soltan Institute for Nuclear Studies, Warsaw, Poland.

6 Also at Massachusetts Institute of Technology, Cambridge, USA.

7 Also at Université de Haute-Alsace, Mulhouse, France.

8 Also at Brandenburg University of Technology, Cottbus, Germany.

9 Also at Moscow State University, Moscow, Russia.

10 Also at Institute of Nuclear Research ATOMKI, Debrecen, Hungary.

11 Also at Eötvös Loránd University, Budapest, Hungary.

12 Also at Tata Institute of Fundamental Research - HECR, Mumbai, India.

13 Also at University of Visva-Bharati, Santiniketan, India.

14 Also at Facoltà Ingegneria Università di Roma "La Sapienza", Roma, Italy.

15 Also at Università della Basilicata, Potenza, Italy.

16 Also at Laboratori Nazionali di Legnaro dell'INFN, Legnaro, Italy.

17 Also at California Institute of Technology, Pasadena, USA.

18 Also at Faculty of Physics of University of Belgrade, Belgrade, Serbia.

19 Also at University of California, Los Angeles, Los Angeles, USA.

20 Also at University of Florida, Gainesville, USA.

21 Also at Université de Genève, Geneva, Switzerland.

22 Also at Scuola Normale e Sezione dell'INFN, Pisa, Italy.

23 Also at INFN Sezione di Roma; Università di Roma "La Sapienza”, Roma, Italy.

24 Also at University of Athens, Athens, Greece.

25 Also at The University of Kansas, Lawrence, USA.

26 Also at Institute for Theoretical and Experimental Physics, Moscow, Russia.

27 Also at Paul Scherrer Institut, Villigen, Switzerland.

28 Also at University of Belgrade, Faculty of Physics and Vinca Institute of Nuclear Sciences, Belgrade, Serbia.

29 Also at Adiyaman University, Adiyaman, Turkey.

30 Also at Mersin University, Mersin, Turkey.

31 Also at Izmir Institute of Technology, Izmir, Turkey.

32 Also at Kafkas University, Kars, Turkey.

33 Also at Suleyman Demirel University, Isparta, Turkey.

34 Also at Ege University, Izmir, Turkey.

35 Also at Rutherford Appleton Laboratory, Didcot, United Kingdom.

36 Also at INFN Sezione di Perugia; Università di Perugia, Perugia, Italy

37 Also at KFKI Research Institute for Particle and Nuclear Physics, Budapest, Hungary.

38 Also at Institute for Nuclear Research, Moscow, Russia.

39 Also at Horia Hulubei National Institute of Physics and Nuclear Engineering (IFIN-HH), Bucharest, Romania.

40 Also at Istanbul Technical University, Istanbul, Turkey. 